Portland State University

PDXScholar

6-2-2021

\title{
Incorporating Conditional ß-Mean based Equity Metric in Coverage based Facility Location Problems
}

Rohan Sirupa

Portland State University

Follow this and additional works at: https://pdxscholar.library.pdx.edu/open_access_etds

Part of the Transportation Engineering Commons

Let us know how access to this document benefits you.

Recommended Citation

Sirupa, Rohan, "Incorporating Conditional ß-Mean based Equity Metric in Coverage based Facility Location Problems" (2021). Dissertations and Theses. Paper 5714.

https://doi.org/10.15760/etd.7587

This Thesis is brought to you for free and open access. It has been accepted for inclusion in Dissertations and Theses by an authorized administrator of PDXScholar. Please contact us if we can make this document more accessible: pdxscholar@pdx.edu. 
Incorporating Conditional $\beta$-Mean based Equity Metric in Coverage based Facility Location Problems

by

Rohan Sirupa

A thesis submitted in the partial fulfillment of the requirements for the degree of

Master of Science

in

Civil and Environmental Engineering

Thesis Committee:

Avinash Unnikrishnan, Chair

Miguel A. Figliozzi

Christopher M. Monsere

Portland State University

2021 
C 2021 Rohan Sirupa 


\begin{abstract}
A classical maximum coverage facility location problem (MCLP) tries to maximize the coverage by serving all possible demands within the specified coverage radius. Depending on the applications, several families of MCLP variants have been developed - each with its unique set of additional constraints. One specific application is locating Emergency medical service (EMS) units. EMS units such as ambulances or drones with emergency supplies are located using models such as the maximum expected survival location problem (MEXSLP). However, a classical MEXSLP model tends to locate EMS units near densely populated regions, resulting in increased response times for lowly populated regions. If equity is to be taken into consideration, the EMS units would be placed more uniformly across the region which might result in reduced overall coverage.
\end{abstract}

In this thesis, a conditional expectation measure called the conditional $\beta$ mean $(\mathrm{CBM})$ is considered and integrated into the facility location models. First, the CBM measure is integrated into the MCLP, resulting in the development of a new MCLP model. Using an alternative and more intuitive definition for CBM, another modified MCLP model incorporated with CBM is developed. These new MCLP models incorporated with CBM are single objective facility location models with flexible coverage radius constraints. Two standard test cases and a Portland Metropolitan region dataset are used to test the MCLP models. A simple greedy based heuristic is developed, and its performance is compared to a state- 
of-the-art Mixed Integer Programming solver Gurobi. The integration of CBM into the MCLP model has improved its coverage. The proposed heuristic provides reliable solutions and saves considerable computational time.

Using the same CBM measure, equity is incorporated into the MEXSLP model. We propose two different approaches to estimate CBM and develop two modified MEXSLP models improving the quality of service provided to outlying and lowly populated areas. The two MEXSLP models modified with CBM are linear, and the decision maker has the leverage to chose the level of equity in the solution using the parameter $\beta$. The same Portland dataset is used for testing the MEXSLP models. Integration of CBM into the MEXSLP has improved the service in Portland suburbs by locating few EMS units closer to them. 
To my family 


\section{Acknowledgements}

This work is a product of the best wishes and support of a lot of people. I will try and do my best to acknowledge everyone.

First and foremost, I would like to sincerely thank Prof. Avinash Unnikrishnan, who has been a mentor in the truest sense. I would like to especially thank him for his continuous guidance, patience, and support towards this thesis and research. He sure is an exceptional advisor, and I can't think of any other person who can inspire me better. I truly feel fortunate for the opportunity I had to learn in an environment with total academic freedom and support, where showing up to every meeting with more questions than answers was met with enthusiasm and encouragement.

I would also like to thank Prof. Miguel Figliozzi and Prof. Christopher Monsere for agreeing to serve on my thesis committee and adding value to this work.

I am very grateful to the National Science Foundation (NSF) for enabling this work through much needed financial support. This research is a product of NSF grants awarded to Prof. Unnikrishnan (Grant numbers: 1562109 and 1826337). I am also thankful for the support I received from the Civil and Environmental Engineering department through the teaching assistantships.

I would like to mention Darshan Chauhan, who is a fellow classmate, labmate, friend, senior, mentor, and almost like a brother. I cannot thank him 
enough for all the motivation, encouragement, support, and guidance he provided at every step, every turn, and every dead-end of this journey.

A special thanks to Dr. Arkamitra Kar for encouraging me to pursue graduate studies and for the fantastic opportunities he has given me. I would like to thank Prof. Sridhar Raju, Dr. Chandu Parimi, and Dr. Anasua Guharay for introducing me to the field of research during my undergraduate studies.

A lot of people enriched my time in Portland. I would like to thank my fellow classmates and friends Santiago, Jaclyn, Mike, Frank, Katherine, and Travis for the mutual exchange of knowledge, fun, laughter, and occasional frustrations with homework and projects. I would also like to thank Dr. Jason Anderson for sharing his knowledge, inspiring me with his work, and showing me the fun in research. Thanks to Sam, Kiley, and Sarah for not just being great staff but helping me through all the administrative hurdles.

Special thanks to my friends Pratik, Pranav, Rushabh, Raghu, Munisha, and Srinivas for being there for me during some of my most challenging times and helping me see the path when I lost my track. Thanks to all other friends at Goose Hollow for all their help, support, evening runs, workouts, bubble tea walks, weekend trips, and all other stuff we cherish from the last two and half years.

Finally, a very big shout out to my beloving family: dad Krishna, mother Sravani, and sister Lahari, who always believed in me, supported me, encouraged 
me, and made sure I was doing fine all through my journey here over my daily calls to India. My love to all my long-distance friends from India who remind me of the fun we had, the hardships we shared, and the time we spent.

A hearty thanks to all others whom I could not acknowledge here but have been a part of this marvelous journey. 


\section{Contents}

Abstract $\quad$ i

Dedication $\quad$ iii

Acknowledgments $\quad$ iv

List of Tables $\quad$ x

List of Figures $\quad$ xi

1 Introduction 1

1.1 Motivation and Background ................. 1

1.2 Conditional $\beta$-Mean . . . . . . . . . . . . . . . . . 3

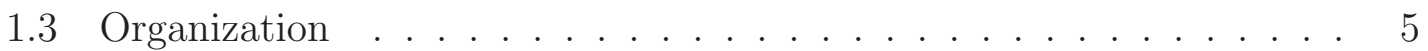

2 Literature Review $\quad 6$

2.1 Facility Location Problems and Models . . . . . . . . . . . . . . . . . 6

2.2 Emergency Medical Services Applications and Models . . . . . . . . . 11

2.3 Equity Measures for FLP . . . . . . . . . . . . . . . . . . 21

2.4 Conditional $\beta$-Mean . . . . . . . . . . . . . . . . 27

2.5 Contributions . . . . . . . . . . . . . . . . . . . . 29

3 Facility Location Problem Application 30 
3.1 MCLP Problem Description . . . . . . . . . . . . . . . . . 30

3.2 MCLP-CBM-I Model . . . . . . . . . . . . . . . . . 31

3.3 MCLP-CBM-II Model . . . . . . . . . . . . . . . . . 38

4 EMS Location Problem Application 45

4.1 MEXSLP Problem Description . . . . . . . . . . . . . . . 45

4.2 MEXSLP-CBM-I Model . . . . . . . . . . . . . . . . . . . . . 48

4.3 MEXSLP-CBM-II Model . . . . . . . . . . . . . . . . . 54

5 Analysis $\quad 59$

5.1 MCLP-CBM Models Analysis . . . . . . . . . . . . . . . . 59

5.1.1 Analysis on Portland Metropolitan Data . . . . . . . . . . 59

5.1.2 Analysis on Standard Test Dataset . . . . . . . . . . . 63

5.2 Greedy Heuristic and Application . . . . . . . . . . . . . . . 65

5.2.1 Heuristic Methodology ............... 65

5.2.2 Heuristic Performance Analysis . . . . . . . . . . . . 66

5.3 EMS Applications/MEXSLP Models Analysis . . . . . . . . . . . . 70

5.4 Summary . . . . . . . . . . . . . . . . . 80

6 Conclusions $\quad 82$

$\begin{array}{lr}\text { Bibliography } & 85\end{array}$

A Appendix: Portland Metropolitan Dataset 96 
B Appendix: Standard P-Median Test Cases 


\section{List of Tables}

5.1 MCLP Models - Objective and Gurobi Gap using PDX Dataset. . . . 62

5.2 MCLP Models - Objective and Gurobi Gap using P-Median Dataset. 64

5.3 Heuristic Results for MCLP-CBM Models using P-Median Dataset . . 67

5.4 Heuristic Results for MCLP-CBM Models for PDX Data . . . . . . . 68

5.5 Heuristic Performance . . . . . . . . . . . . . . . . . 70

5.6 MEXSLP Models - Objective and GAP Values. . . . . . . . . . . . 71

5.7 MEXSLP Model Travel Time Statistics. . . . . . . . . . . . . . . . . 77

5.8 MEXSLP-CBM-I Model Travel Time Statistics. . . . . . . . . . . . . 77

5.9 MEXSLP-CBM-II Model Travel Time Statistics. . . . . . . . . . . . . 78 


\section{List of Figures}

5.1 Demand points and potential facilities in the Portland Metropolitan Region. . . . . . . . . . . . . . . . . . 60

5.2 MEXSLP solution $(p=20) \ldots \ldots \ldots \ldots$. . . . . . . . . . . . . . .

5.3 MEXSLP CBM-I and MEXSLP CBM-II solutions $(p=20$ and $\beta=1 \%) \ldots . . .73$

5.4 MEXSLP CBM-I and MEXSLP CBM-II solutions $(p=20$ and $\beta=20 \%)$. Size of star corresponds to EMS units at that location. . . . . . . . . . . . . . . . 74

5.5 MEXSLP solution $(p=40) . \ldots \ldots \ldots$. . . . . . . . . . . 74

5.6 MEXSLP CBM-I and MEXSLP CBM-II solutions $(p=40$ and $\beta=1 \%)$. . . . 75

5.7 MEXSLP models solutions $(p=40$ and $\beta=20 \%$ ). Size of star corresponds to EMS units at that location. . . . . . . . . . . . . . 76

5.8 Distribution of Travel Times from facilities to demand $(p=20$ and $\beta=20 \%) \quad$. . 79 


\section{Introduction}

\subsection{Motivation and Background}

The Maximum Covering Location Problem (MCLP) was first introduced in Church and ReVelle (1974). Since then it has been popular and paved path for many problems to locate facilities for public and private services, such as postal, police, ambulances, and warehouses. Later, Current and Storbeck (1988) proposed the Capacitated Maximum Covering Location Problem (CMCLP) where each open facility has a specific capacity, and the total demand assigned to any facility cannot exceed this capacity. The structure of such a problem would only allow covering the demand points within the coverage radius, and anything outside will be left unserved. This approach seems reasonable for many systems, but there is no such hardbound on the coverage radius for public services, such as postal, medical, or police. They are available to serve the entire jurisdiction. Coverage radius is a tool aiming to provide a minimum service level to the largest number, but not to restrict its services.

One of the major applications of MCLP models is in finding optimal sites to places the EMS units such as ambulances. In these models, the facility locations are chosen such that the response times are minimum. Response time is the time 
needed for an EMS unit to arrive at the patient after the emergency call is made. Li et al. (2011) provides a brief study of such covering models for EMS.

One strong assumption made in these EMS covering models is that the patient is considered uncovered if it is not within the coverage area, i.e., if a patient cannot be reached within the predetermined response time threshold by any EMS unit, then the patient is left out as uncovered in the model. Nevertheless, even if a patient is located very far, it would be served by the closest available EMS unit. Even if an EMS unit arrives in time less than the response time threshold, there is no guarantee that a patient will survive. It has always been a probability; the shorter the response time greater is the probability of survival.

This idea helped develop the Maximum Survival Location Problems (MSLP) and Maximum Expected Survival Location Problems (MEXSLP) models, where the objective is to maximize the aggregated sum of the survival probability of all demand sites. Erkut et al. (2008) explains the application of various survival location problems.

The objective of most of the research on EMS location problems has been to maximize the demand served, i.e., to help as many people as possible using available resources. However, sometimes it might be reasonable to consider solutions other than the one with the maximum number of people being served on time. For instance, with the current objective, the model is inevitably forced to allocate resources closer to the densely populated areas, resulting in longer response times 
for patients in low-density or remote areas. This is an efficient way of utilizing resources, but the question is whether the solution is equitable.

Intuitively, if everyone gets service within the same response time, the solution would be fair and equitable. However, achieving the same level of service for remote areas as we get for the densely populated urban areas requires a much higher volume of resources. So, providing the same level of service for everyone might not be truly fair. A genuinely fair solution is achieved by having a balance between efficiency and equality. We chose the conditional $\beta$-mean measure for obtaining equity, and in the next section, we go through more details about this measure.

\subsection{Conditional $\beta$-Mean}

To capture fairness or equity among the customers or facilities, we consider the conditional $\beta$-mean measure proposed in Ogryczak and Zawadzki (2002). The conditional $\beta$-mean measure is closely related to the concept of Conditional Valueat-Risk (CVaR), which has been popularly used in financial optimization problems (Rockafellar et al., 2000), and recently even in various managerial and engineering problems (Filippi et al., 2020). If the outcomes are from a discrete probability distribution, the conditional $\beta$-mean corresponds to be the $\mathrm{CVaR}$ of this distribution with parameter $\beta$. The application of conditional $\beta$-mean in this work is inspired from Filippi et al. (2019a), and it is defined is as follows: 
Definition: Given a $\beta \in(0,1]$, the conditional $\beta$-mean measure is defined as the average of the highest or lowest $(100 * \beta) \%$ outcomes of demand taken into account.

Depending on whether the highest or lowest outcomes are being considered, we get the worst-case or best-case for conditional $\beta$-mean. When the parameter $\beta$ is 0 , the conditional $\beta$-mean is the highest or lowest outcome, and when $\beta$ is 1 , the corresponding conditional $\beta$-mean is the same as a standard mean. In the case of discrete problems, if there are $m$ possible outcomes and $\beta=k / m$, then conditional $\beta$-mean represents the mean of the highest $k$ outcomes, which is similar to the $k$-centrum solution concept.

Intuitively, by definition of conditional $\beta$-mean, maximizing or minimizing this measure would shorten the tail in the distribution of outcomes and reduce the extremities. So, conditional $\beta$-mean can be used to capture equity by reducing dispersion in outcomes.

Conditional $\beta$-mean has the following properties, which makes it suitable for application of equity and fairness concepts in facility location problems, (Filippi et al., 2019b)

i. This measure is a Schur-Convex function, thus making it a suitable inequality measure.

ii. By definition, it acts as an upper bound on the standard mean of outcomes. 
iii. Can be implemented in Mixed Integer Linear Programs (MILP) models with a limited number of constraints and variables.

\subsection{Organization}

The remaining part of the thesis is organized as follows: Chapter-2 reviews the relevant literature related to facility location problems, emergency medical services location problems, equity measures in facility location problems, and application of conditional $\beta$-mean. Chapter-3 provides the problem description and formulations of two maximum covering facility location models incorporating conditional $\beta$-mean measure. Chapter- 4 provides the problem description and formulations of two emergency medical service location models incorporating conditional $\beta$-mean measure for equity purposes. Chapter- 5 examines the performance of the developed conditional $\beta$-mean models with other regular models using a state-of-the-art Mixed Integer Programming (MIP) solver. A simple heuristic is also proposed in this chapter to solve two of the proposed models. A sensitivity analysis is performed to estimate the impact of the conditional $\beta$-mean constraint and the impact of other parameters on objective functions. The final chapter, chapter-6, provides the summary of this work and scope of future directions for research. 


\section{Literature Review}

This chapter reviews relevant literature on the following topics: (i) facility location problems, (ii) location model applications in Emergency Medical Services (EMS), (iii) papers focusing on modeling equity metrics in the facility location problems, and (iv) facility location models which have integrated conditional $\beta$ mean equity measure.

\subsection{Facility Location Problems and Models}

The goal of the Facility Location Problem (FLP) is to identify locations to open facilities to satisfy customer demands and optimize specific objectives. The facility type can vary from storehouses, postal services, emergency services, nuclear power plants, garbage disposal sites, etc. Depending on the application, the objectives and constraints of the FLP will be different.

The FLP can be solved for public or private sector services. For a private sector facility location analysis, it is reasonable to assume that the model's objective is to minimize the total costs involved in the system, which primarily includes set-up, distribution, and maintenance costs. In contrast, the public sector facil-

ity location problems are more concerned with the quality or level of service. An 
example of an objective for public sector applications is maximizing the demand coverage.

Toregas et al. (1971) and Toregas and ReVelle (1972) introduced the Location Set Covering Problem to find the minimum number of facilities required to cover all users within the desired service distance, which is also called the service or coverage radius. If there is a limitation on the number of facilities, achieving complete coverage is not possible. For such cases, Church and ReVelle (1974) developed the Maximum Covering Location Problem (MCLP), whose objective is to attain the highest coverage by maximizing the population being covered within the service radius.

Church and ReVelle (1974) also includes another model called MCLP with mandatory closeness constraints, which focuses on covering as many people as possible within the coverage radius, and shows concern for the quality of service being provided for people outside the coverage radius, $S$. In short, the model covers the maximum population possible within the coverage radius but also ensures that everyone has at least one facility in a distance $S^{\prime}$, called the mandatory coverage radius. This is an approach to make the solution fairer for every person in the system. Lower the difference between $S$ and $S^{\prime}$, the fairer solution is to people outside coverage radius.

Another important issue in locating both public or private facilities is the capacity. In most cases, any open facility can only handle a certain amount of 
demand, leading to constraints on their capacity or workload. Considering this, a new model called the Capacitated MCLP was proposed in Chung et al. (1983) and Current and Storbeck (1988) with capacitated facilities. These models are an extension of the maximum covering models with additional capacity constraints.

Pirkul and Schilling (1991) further extended the Capacitated MCLP model by providing backup service to users. Pirkul and Schilling (1991) presents the formulation and solution procedure for this model, where all facilities are capacitated, and every demand has a primary and secondary backup facility. Pirkul and Schilling (1988) considered a similar problem of allocating capacitated facilities with backup service providers, but instead of maximizing the coverage, their objective was to minimize the average distance traveled.

Haghani (1996) presents two different formulations and two different solution procedures for capacitated MCLP models. The first formulation is a mixedinteger linear programming model. The second formulation aims to maximize the weighted covered demand while minimizing the average distance between uncovered demands and open facilities. By which, this formulation tries to accommodate the uncovered demands with facilities having excess capacity.

Albareda-Sambola et al. (2009) introduces the Capacity and Distance Constrained Plant Location Problem (CDCPLP), an extension of the discrete capacitated facility location problem. This model has constraints on facility capacity and maximum distance traveled by the vehicles. First is the limited capacity of a 
facility to serve demands from customers. Next is that each vehicle can only travel a limited distance to complete their assigned facility-customer-facility round trips. CDCPLP model identifies the facilities to open, finds the number of vehicles required at each facility, and assigns each customer to a specific facility and vehicle to minimize all costs involved while not violating the facility capacity or vehicle distance constraints. CDCPLP is similar to the capacitated facility location problem, but it includes fleet management.

With the recent development of Unmanned Aerial Vehicles (UAVs) or drones, several companies like Amazon, Google, and UPS are evaluating their potential for use in commercial delivery services. Drones are not restricted by the availability of existing infrastructure and not affected by traffic, making them suitable for emergency services, such as delivering critical medical supplies. With such applications in mind, FLP models incorporating drones have been developed and studied. Chauhan et al. (2019) proposed a maximum coverage facility location problem with drones (MCFLPD) model, a complicated version of MCFLP as it has additional drone constraints range, availability, and payload. MCFLPD model is similar to the CDCPLP model in Albareda-Sambola et al. (2009), while CDCPLP focuses on minimizing the cost, MCFLPD focuses on maximizing the coverage. Also, CDCPLP assumes a fixed number of drones in each facility, while MCFLPD assumes a fixed number of drones in the entire system, which gives a new drone allocation feature and adds more complexity to the problem. In 
Chauhan et al. (2019), the model is not based on one-to-many deliveries in a trip. It is assumed that all deliveries are one-to-one, i.e., they leave from a facility, complete the delivery and come back to the same facility.

Most of the FLP models discussed so far are static and deterministic does not account for uncertainty in the system. The system's uncertainty could be dealt with either by optimizing the expected scenario or optimizing the worst-case scenario. Sheppard (1974) was among the first to use scenario planning in facility location. This model minimizes the expected cost across all scenarios. Schilling (1982) suggests an approach where the initial decisions implemented are the ones that are common in most scenarios and delay other decisions till uncertainty is not resolved. Snyder et al. (2007) states that scenario planning allows decisionmakers to model dependence among random parameters, making it suitable for uncertainties in FLP. Snyder et al. (2007) studies the stochastic facility location problem with risk pooling, which locates distribution centers while minimizing the total fixed location costs, transportation costs, and inventory costs.

Besides this scenario planning, uncertainty can also be captured using a measure called $\alpha$-reliable regret. Daskin et al. (1997) presented a model called the $\alpha$-reliable minimax regret and combined it with a p-median model. In many situations, minimizing the $\alpha$-reliable maximum regret is appropriate compared to any average or worst-case regret. However, computationally, it is not easy to solve and obtain. So, Chen et al. (2006) proposes a new model called $\alpha$-reliable 
mean-excess regret or mean-excess model. This measure explicitly accounts for the magnitude of regrets in the distribution's tail, making it computationally much easier to solve or apply over the $\alpha$-reliable minimax model.

Ghosh and McLaerty (1982) proposes a model where the sum of regrets is minimized over all possible scenarios. The objective of minimizing the sum of regrets is equivalent to minimizing the expected regret with all scenarios having the same probability.

There is a significant amount of work in FLP and several extensive reviews of various FLP variants. ReVelle and Eiselt (2005), Revelle et al. (2008), and Daskin (2011) provide a detailed review of various FLP variants and associated formulations and solution methods. Farahani et al. (2012), Berman et al. (2010), and Schilling (1993) provide an exhaustive review of MCLP variants. For the FLP under uncertainty, an in-depth review is provided by Berman and Krass (2001);

Correia and Saldanha-da Gama (2019); Snyder et al. (2016) and Snyder (2006).

\subsection{Emergency Medical Services Applications and Models}

The concept of coverage is an appealing measure for the performance of facility location problems, especially in locating Emergency Medical Services (EMS), where the quality of service is crucial. An important factor pertaining to the quality of service in any EMS system is timeliness in providing these services. 
Most of the EMS locating models are variations of the classical MCLP introduced in Church and ReVelle (1974). So, in other words, most of the covering models discussed in the previous section can be modified to the requirements of an EMS system. This MCLP model assumes that the vehicles are always free and doesn't include any adjustments for vehicles such as ambulances being busy. Considering that ambulances might be busy serving other users, Daskin (1983) proposed an extension of the MCLP model called the Maximal Expected Covering Location Problem (MEXCLP). MEXCLP assumes that the probability of an ambulance being busy is the same across the system. Recognizing that this might not be the case, other location models have been developed. Batta et al. (1989) modified the MEXCLP model and introduced the Adjusted MEXCLP (AMEXCLP), which recognizes that servers do not operate independently they might have different busy probabilities depending on the location.

In emergency service systems queuing is undesirable. When a service request occurs, the nearest ambulance is dispatched. Assume another request arrives at the same facility before the return of the ambulance. Now instead of forming a queue at this facility, an ambulance should be sent from the next closest facility having resources. Though both the MEXCLP and AMEXCLP have considered the possibility of an ambulance being busy, neither of them addressed the stated issue.

This need for a backup facility is important in regions/zones with heavy 
demand or population. An effective way of solving such a problem would be providing a backup facility for each demand point along with an assumption of a reasonable threshold on each facility's workload.

Berlin (1974) is one of the earliest works on locating facilities with backup services. They first used a sequential approach on a location set covering problem (Toregas and ReVelle, 1972) to find the minimum number of ambulances and their dispatching sites. Then using a simulation model estimated the utilization of each ambulance. Daskin and Stern (1981) proposed another model by modifying the set covering problem using a hierarchical multi-objective formulation which minimized the required number of facilities and maximized the number of times demand is covered at the same time. Ruefli and Storbeck (1982) created another hierarchical maximum covering model where demands are covered by primary facilities, and secondary facilities provide backup to the primary facilities.

Hogan and ReVelle (1986) developed a coverage maximizing model where each demand zone is assigned with two facilities, a primary facility and another secondary backup facility if the primary is busy serving another. Pirkul and Schilling (1988) and Pirkul and Schilling (1991) also considered a similar model providing backup services while assuming that all facilities are capacitated. Narasimhan et al. (1992) proposed an extension of such a model to provide multiple levels of backup services.

Another approach for ambulance or EMS location problems is using multiple 
objectives. Daskin et al. (1988) integrated various covering models, including multiple excesses, backup, and expected coverage models. This multi-objective model is a reformulated version of the hierarchical objective model in Daskin and Stern (1981) and allows the decision-maker to have a trade-off between extra coverage and the number of facilities.

Araz et al. (2007) proposed a multi-objective facility covering location model based on the models provided in Hogan and ReVelle (1986) and Pirkul and Schilling (1988). Their model consists of three objectives: (i) maximize the demand covered by a single vehicle, (ii) maximize the demand with a backup service, (iii) minimize the total distance from facilities located farther than a specified distance standard for demand points. They proposed to solve this model using a fuzzy goal programming approach.

In the literature, there are other works which consider different coverage assumptions regarding, such as allowing partial coverage or even restrict coverage, which include Revelle et al. (1996), Berman and Krass (2002) and Karasakal and Karasakal (2004). However, these models aren't discussed in detail here as they do not focus on maximizing the coverage. Brotcorne et al. (2003) can be referred for a more detailed review of literature on the ambulance or EMS location problems.

All the EMS location models discussed so far do not consider the possibility of having multiple types of EMS units or levels of severity in a patient's condition. Batta et al. (1989) studied one such EMS model considering multiple casualty 
types with probabilistic travel times, but with just one type of server, i.e., the EMS unit.

In literature, certain studies focus on two-tiered EMS systems considering two types of EMS units: Basic Life Support (BLS) and Advanced Life Support (ALS). The BLS units are equipped with basic equipment, whereas the ALS units are equipped with life-saving procedures, including the equipment provided in BLS units.

Bélanger et al. (2019) summarize and discusses the modern approaches in EMS modeling, addressing the problems related to EMS fleet management, EMS vehicle location or relocation, and their dispatching decisions. Their work begins with the literature on static ambulance location models followed by multi-period relocation models, dynamic relocation models, and approaches related to ambulance dispatching decisions.

Schilling et al. (1979) proposed two optimization models (TEAM and FLEET) to maximize the fraction of demand covered by both the BLS and ALS units in the system. ReVelle and Marianov (1991) and Marianov and ReVelle (1992) extended the FLEET model to maximize the coverage provided by both EMS units while ensuring individual and joint reliability requirements.

Mandell (1998) proposes a probabilistic covering model, which unlike Batta et al. (1989), considers multiple types of medical units but not multiple casualty types. This model considers both BLS and ALS units but assumes a patient 
or call is covered if and only if an ALS unit can respond within a prespecified amount of time.

Marianov and Serra (2001) introduce another model that maximizes the coverage considering a limited number of BLS and ALS EMS units in a hierarchical system with congestion. Here, demand is considered covered when both types of units are within the prespecified distance and do not have to wait in the queue with more than a fixed number of demands. Both the models proposed in Mandell (1998) and Marianov and Serra (2001) assume two types of EMS units, but they do not consider multiple types of demands.

McLay (2009) extended the MEXCLP proposed in Daskin (1983), introducing the Maximum Expected Coverage Location Problem with two types of servers (MEXCLP2) to optimally locate two types of EMS units while serving multiple types of customers. In MEXCLP2, the ALS units are non-transport quick response vehicles, and BLS units are ambulances with the ability to transport patients. Here, for every call, both types of units are dispatched so that the ambulance can transport the patient to the hospital after ALS units provide the necessary treatment.

Next, Boujemaa et al. (2018) aims to account for the inherent uncertainty from demands and designs a robust two-tiered EMS system. This study proposes a two-stage stochastic location-allocation model that simultaneously determines EMS units' location, the type and number of units to be dispatched, and the 
demands covered by each EMS station.

Grannan et al. (2015) developed a military medical evacuation (MEDEVAC) system to respond and transport casualties based on their severity using two types of air ambulances. They proposed a binary linear programming model to locate air assets and create response districts using a preferred dispatch list. This model defines three categories for casualties and aims to maximize the proportion of highest priority casualty responded to within a prespecified response time threshold while not violating the performance constraints on other types of casualties.

Gendreau et al. (1997) proposed a model called the double standard model (DSM), which determines the location of EMS vehicles while maximizing the proportion of demand covered twice within a predefined distance. Later, Liu et al. (2014) and Liu et al. (2016) extended this DSM model considering multiple types of EMS vehicles and demands with various priority levels.

Gendreau et al. (2001) proposed an ambulance relocation model accounting for the dynamic nature of the EMS systems. This relocation problem is developed on the DSM problem, proposed in Gendreau et al. (1997), by adding a penalty term in the objective function, which takes in the cost of relocation of vehicles.

Mason (2013) also proposed a dynamic ambulance relocation model named the real-time multi-view generalized cover repositioning model (RtMvGcRM). Like Gendreau et al. (2001), RtMvGcRM's objective is to maximize the quality of service while minimizing the relocation costs. However, RtMvGcRM considers 
multiple types of ambulances having varying performance in the system.

Van Barneveld et al. (2017) developed a model for an EMS system in the Netherlands with two types of units: Rapid Responder Ambulances (RRA) and Regular Transport Ambulances (RTA). Among the two, RRA units are faster, but they cannot transport a patient. They use compliance tables to identify the desired locations to place EMS units considering they get busy. They extend the ambulance relocation model from Gendreau et al. (2006) and the MEXCLP2 model from McLay (2009) to propose an integer linear programming model computing these compliance tables.

Yoon et al. (2021) propose a two-stage stochastic programming model that determines how to locate and dispatch different types of EMS units. This model is called the Scenario-based Ambulance Location for Two types (SALT) model, considering two types of EMS units, ALS and BLS units, and two types of callpriorities, high and low. High-priority calls are assumed to be life-threatening and require ALS units, whereas, for low-priority calls, either BLS or ALS units are sufficient. They proposed a data-driven approach of sampling call arrival scenarios directly from the call logs to avoid distributional assumptions and capture the Spatio-temporal correlation between calls.

Besides all these, Hammami and Jebali (2019) propose a two-tiered EMS model to determine the location and modular capacity of ambulance stations that minimize the total cost while respecting the predefined response time threshold. 
Their approach considers advanced trip information such as total service time, busy fraction, and modular capacity of base stations. Compared to other traditional approaches, this approach is unique in that it considers the travel time from the demand to the trauma center in the total service time. However, unlike other EMS models, this model focuses on minimizing cost instead of maximizing the total coverage.

Besides the ambulance stations, an EMS system also contains trauma care centers. Now we will discuss models which identify the locations of both the EMS stations and trauma centers. Branas and Revelle (2001) proposed the Trauma Resource Allocation Model for Ambulances and Hospitals (TRAMAH), which is the first of its kind to locate both trauma centers and air ambulance depots. This model has the computational flexibility to locate both these assets as either separate resources or in tandem. TRAMAH can be used to develop an EMS system from absolute nothing or to improve an existing system.

Lee et al. (2012) present a mathematical model and its solution methodology to identify the optimal location for trauma centers and air ambulances. The model proposes a method that uses integer programming and simulation to update busy fractions in the model iteratively.

Cho et al. (2014) also studied the problem of simultaneously locating trauma centers and helicopters. In their work, they endogenize the calculation of the helicopter's busy fractions into the optimization problem. Later, Lee and Jang 
(2018) extended this model into a multi-period model by introducing another aspect: when to locate. However, the original model is quite challenging to solve, and the addition of time-dependent parameters makes it much more difficult.

Jayaraman and Srivastava (1995) also proposed a model that tries to maximize the expected coverage of the demands while simultaneously locating facilities and allocating multiple types of equipment to them.

After locating EMS units, the next most important question is how to dispatch these EMS units in real-time? Bandara et al. (2012), Sudtachat et al. (2014), Yoon and Albert (2018), and Yoon and Albert (2020) have answered this problem using the Markov Decision Process (MDP) which dynamically determines which EMS unit to be dispatched using real-time system status. These dispatching policies are developed to maximize patients' survival probability by incorporating the arrival call's degree of urgency. The optimal policy provides an ordered preference list of EMS units to dispatch based on priority.

It is to be noted that, even though all the above-mentioned models have a different approach to estimate coverage and provide better quality of service, all of them have a primary objective of maximizing coverage, i.e., capture the maximum population possible within the predefined service radius. Implementing this approach alone can bring disparities among the users regarding access to public services, especially among rural and urban communities. In the following section, we discuss literature on how such disparities were answered using equity 
measures.

\subsection{Equity Measures for FLP}

With a single objective of maximizing coverage, models tend to locate facilities closer to urban areas having a dense population, leading to adverse outcomes in rural areas. Even if more EMS units are made available, these models tend to concentrate units in favor of the urban population unless the coverage is closer to $100 \%$.

Li et al. (2011) states that the literature has thoughtfully investigated the efficiency of EMS facility location, but the solutions' equity has been overlooked. Aringhieri et al. (2017) says that "equity is one of the most challenging concerns in the healthcare sector, like EMS systems, as it evaluates the fairness of how resources are allocated to patients". According to Oliver and DeSarbo (1988), fairness is the perception of customers on how the ratio of their outcomes to inputs is comparable to the other parties in the system.

The words "equity" and "fairness" are often used interchangeably in the existing literature and mean the same. However, the words "equality" and "equity" necessarily might not mean the same. In general, equality means providing every individual with the same amount of resources or opportunities. Whereas equity recognizes the difference in circumstances of every individual and provides resources as needed to achieve the same outcome. 
The MCLP with mandatory closeness constraints proposed in Church and ReVelle (1974) can be considered as an attempt to provide a fairer solution for uncovered demand points. As stated in this model, the closer the service and coverage radii, the fairer the solution is to people outside the coverage radius. Pirkul and Schilling (1991) also provides a similar extension for their capacitated MCLP model considering a maximal response or service distance, which is meant to be a tool to ensure a minimum level of service but not to hold back any resources. However, their results state that improved service to uncovered demands is attained only at the cost of reduced coverage, i.e., there is always a trade-off between total service and total coverage.

In some EMS location problems, equity has been evaluated indirectly by choice of model. One way of ensuring fairness in outcomes is by adding chance or reliability constraints that enforce a minimum level of service throughout the system (Ball and Lin, 1993; ReVelle and Hogan, 1989). However, such indirect approaches neither evaluate equity explicitly nor examine the interaction between equity and efficiency measures.

Maximin modeling is a popular approach to incorporate equity, where the objective is to maximize the level of service received by customers hardest to get. This approach has been used in various research efforts usually as bi-objective models (Chanta, 2011; Current et al., 1990; Erkut and Neuman, 1992; Kostreva et al., 2004; Zhan and Liu, 2011). These bi-objective models are often formulated 
by combining one efficiency measure and another equity measure. However, these studies do not prescribe anything about weighing the two objectives. Instead, they provide a range of options for the decision-maker to choose from. This approach yielded poor performance of the system, which is its major drawback.

Chanta et al. (2014a) considers a bi-objective model whose primary objective is to maximize the expected coverage while the secondary objective is to improve fairness regarding access to rural areas. Chanta et al. (2014a) proposes three alternatives for the secondary objectives, which are: (i) minimizing the maximum distance between every uncovered demand and the open facility closest to it, (ii) minimizing the number of rural demand points that are uncovered, and (iii) minimizing the total number of uncovered demand points, this includes both urban and rural areas. Their results indicate that the model with the first alternative as a secondary objective dominates the other two alternatives.

Savas (1978) broadly describes four principles for evaluating equity in public services, which are equal payments, equal outputs, equal inputs, and equal satisfaction, and explains how each principle can be selected and implemented. Marsh and Schilling (1994) provides a comprehensive review of twenty equity measures in facility location problems, focusing on differences in service outcomes. They compare functional forms of equity and not how these forms can lead to various decisions in reality. They observe and state that there is not much consensus on the best way or suitable way to measure equity. Barbati and Piccolo (2016) 
considers ten popular equity measures, introduces few new properties associated with these measures that could better describe their behavior in the context of optimization, and performs computational analysis to verify if these properties are satisfied or not by ten selected measures. Some of the measures considered in Marsh and Schilling (1994) and Barbati and Piccolo (2016) are: Center (CEN), Range (RG), Mean Absolute Deviation (MAD), Variance (VAR), Maximum Deviation (MD), Absolute Deviation (AD), Summation of Maximum Absolute Differences (SMAD), Schutz's Index (SI), Coefficient of Variation (CV) and Gini coefficient (GC). These measures are formulated such that the level of inequality in the distribution of resources is captured, i.e., lower the value, fairer is the solution. Most of these measures capture various characteristics of the distribution of travel times or distances. Most of these measures do not satisfy the principle of Pareto efficiency that it is possible to improve fairness by making some individuals worse-off. Optimizing fairness measures not obeying Pareto efficiency can lead to locating facilities far away from customers (Barbati and Piccolo, 2016; Filippi et al., 2019a). The following example can help us understand this. Consider three customers located on a straight line, now if we plan to open a facility to serve all of them, intuitively, the fairest location to open would be at infinity.

Following are some of the studies that have implemented the above measures to achieve fairness. López-de-los Mozos and Mesa (2001) studied the MAD measure and its properties when implemented in facility location problems. Drezner 
et al. (2009) analyzed the Gini coefficient's application, as an equity measure, in a facility location problem with uniformly distributed demand points. Ogryczak (2000) formulated a similar model which minimizes the mean distance and MAD measure. Ohsawa et al. (2006) proposes a model where efficiency is sought by optimizing the sum of squared facility-user distances, while equity is measured using the sum of absolute differences of distances. Lejeune and Prasad (2013) solved a tree network facility location model by formulating the median objective as an efficiency measure and the Gini coefficient as a measure for equity.

Few equity measures that gained popularity later are envy, quintile share ratio, and conditional $\beta$-mean. Espejo et al. (2009) applies the concept of envy in an uncapacitated discrete facility location problem. This model attempts to locate $\mathrm{p}$ facilities with the objective being to minimize the total envy experienced by all demand sites. Total envy is defined as the sum of absolute deviations from the general preferences expressed by all demand sites. Chanta et al. (2011) locates facilities in an EMS system by minimizing the total envy of all demand points, where envy is defined as the dissatisfaction of a demand point with respect to the other points. Chanta et al. (2014b) proposes a similar facility location problem with the objective being to minimize envy, which is evaluated using a survival function while having a constraint on minimum survival rate. Rey et al. (2018) applies the concept of envy in the context of humanitarian logistics to collect and redistribute surplus perishable food for hunger relief. 
Drezner et al. (2014) uses the Quintile Share Ratio as an objective function in facility location analysis. There the Quintile Share Ratio is calculated as the ratio of the bottom quintile to the top quintile. The bottom quintile is defined as the total cost paid by the $20 \%$ of demand paying the lowest per-unit cost. Similarly, the top quintile is defined as the total cost of the $20 \%$ demand paying the highest per-unit cost. The denominator of the objective in Drezner et al. (2014) is what we define as CBM with $\beta=0.2$, i.e., Conditional 0.2-Mean.

In the literature, the concept of equity is also adapted with references other than distribution of distances, such as distance between pairs of facilities, maximum demand assigned to a facility, total demand assigned to a single facility, and more. Baron et al. (2007) considered a facility location problem with the objective being to minimize the maximum demand assigned to every facility in the system. Similarly, Berman and Huang (2008) solved for a facility configuration that minimizes the maximum total demand assigned for a facility on the network. Marín (2011) addressed a discrete facility location model which tries to balance the difference between the maximum and minimum demand sites allotted to each facility.

Espejo et al. (2009) proposed a unique discrete facility location problem in which users provide a preference order on potential sites to open facilities. This is an approach aiming to minimize the total envy experienced by users. Prokopyev et al. (2009) proposed a problem called the equitable dispersion problem, whose 
objective is to minimize the range and MAD of the distribution of distances between pairs of open facilities. McLay and Mayorga (2013) discuss the distinction between equity from a server and patient perspective.

Jagtenberg and Mason (2020) discussed how to place ambulances across a region in a fair way, using a fairness measure called Bernoulli-Nash Welfare Function. The classical ambulance location models aim to improve the system's efficiency by maximizing the overall performance, which contrasts with fairness. The most efficient ambulance configurations benefit people living in cities at the expense of people living in rural/remote areas. These fairness measures provide a solution that has a more balanced performance or service across the region/system. Their model also involves various performance concepts such as survival function and the probability of ambulance arrival within the response time threshold. Jagtenberg and Mason (2020) focused on providing the decision-maker with a model that results in one quite fair solution instead of a Pareto frontier.

\subsection{Conditional $\beta$-Mean}

In our study, the fairness or equity measure incorporated with the facility location problems is Conditional $\beta$-Mean (CBM). In this section, we briefly discuss the literature related to CBM.

$\mathrm{CBM}$ is a measure that can capture both the performance and fairness of a system. CBM was proposed in Ogryczak and Zawadzki (2002) as a possible fair- 
ness measure in facility location problems. CBM is related to CVaR, which is a quite popular risk measure in financial optimization Rockafellar et al. (2000). Technically, minimizing CBM is similar to minimizing CVaR over a discrete distribution. CVaR has gained popularity in engineering contexts in recent days (Filippi et al., 2017).

Chapman and Mitchell (2018) applies a fairness measure inspired from CVaR in the context of humanitarian logistics. Their objective function includes three terms, the weighted average of the fixed opening costs of facilities, CVaR of traveling costs, and the average traveling costs for customers to reach facilities. Here, the traveling distances from customers to facilities are known and fixed, i.e., there is not any uncertainty. So, the CVaR calculated here is what we defined as CBM. However, the constraints used to calculate CVaR in their work are nonlinear. In Drezner et al. (2014), the denominator of the Quintile Share Ratio objective calculates the total cost paid by the $20 \%$ demands paying the highest to get served. By definition, the mean of this value is equivalent to $\mathrm{CBM}$ with $\beta=0.2$, i.e., Conditional 0.2-Mean.

Filippi et al. (2019a) defined and analyzed a new problem called the Fair Single Source Capacitated Facility Location problem (F-SSCFLP). This problem is represented as a bi-objective model where the objectives are to minimize total costs and improve fairness. In their work, the CBM is used as the fairness measure and is defined as the average cost paid by the $\beta \%$ customers who have to 
travel the most to reach the nearest facility. Filippi et al. (2019a) analysis states that CBM is more flexible than a classical minimax objective problem, and performs better than MAD or Range measures. Jagtenberg and Mason (2020) also states that CBM to be a potential alternative for maximin models.

\subsection{Contributions}

Unlike the existing works where CBM is incorporated using a bi-objective function, here, CBM is incorporated as a constraint with single objective function. Existing works on incorporating CBM in facility location models focus on minimizing cost whereas this thesis is concerned on maximizing coverage. As CBM is calculated for each open facility, the proposed MCLP models maintain equity concerning facilities. An alternative approach to estimate CBM is proposed, assuming this approach to be faster to solve and intuitively easier to understand. A greedy based heuristic is proposed in this work, and its performance is compared to a state-of-the-art Mixed Integer Programming solver.

The existing MEXSLP models to solve EMS location problems in Erkut et al. (2008) and Jagtenberg and Mason (2020) are nonlinear. Here, we propose two linear MEXSLP formulations, where equity is incorporated in MEXSLP model using CBM. In these EMS location models, the level of equity can be chosen by the decision maker using the parameter $\beta$. 


\section{Facility Location Problem Application}

This chapter focuses on incorporating CBM into the maximum covering facility location problem (MCLP). First, a classical MCLP with capacitated facilities is explained. Next, the concept of CBM is integrated into the classical MCLP as a constraint and a new model called MCLP-CBM-I is developed. Lastly, by proposing an alternate way of defining and calculating CBM, another variant of the MCLP called MCLP-CBM-II is developed.

\subsection{MCLP Problem Description}

In the classical maximum coverage facility location problem (MCLP), we consider a set $I$ with all demand points, and a set $J$ with all potential locations to open facilities. The primary objective of this model is to find optimal locations to open facilities such that maximum possible demand is served while satisfying the constraints on facility's capacity and distance.

In this problem, sometimes, certain facilities may be associated with more costs, in terms of travel distance or travel time, only to satisfy a little more demand. Here we use the concept of CBM, to reduce the disparity among the distances traveled from open facilities to demand points they serve. We try to 
achieve this objective by limiting the CBM of the distances from a facility to its demand points to be less than the facility's coverage radius.

\subsection{MCLP-CBM-I Model}

This MCLP-CBM-I model is a modified version of a capacitated MCLP, incorporated with CBM on facilities. Here, the CBM for each facility is calculated as the average distance of the farthest $\beta \%$ demands served by it. In a capacitated MCLP, any open facility cannot serve demand points outside its coverage radius. In MCLP-CBM-I, it is assumed that the CBM of a facility cannot exceed its coverage radius, allowing the model to utilize a facility's total capacity and serve demands that are close-by but outside the coverage radius. The nomenclature of MCLP-CBM-I model is provided below and we start with formulation MODEL$1 \mathrm{~A}$.

\section{Nomenclature}

Sets

I Set of all demand points

$J \quad$ Set of all potential facility locations

Indices

$$
\begin{aligned}
& i \in \mathrm{I} \\
& j \in \mathrm{J}
\end{aligned}
$$

Parameters 
$d_{i j} \quad$ Distance between demand point $i \in I$ and facility location $j \in J$

$p \quad$ Maximum number of facilities that could be opened

$U_{j} \quad$ Capacity of facility location $j \in J$

$w_{i} \quad$ Demand of point $i \in I$

$r_{j} \quad$ Coverage radius of facility at location $j \in J$

$\beta \quad$ Percentile of demand to be used while estimating CBM

Variables

$x_{i j} \quad 1$, if demand point $i \in I$ is served by facility $j \in J ; 0$, otherwise

$y_{j} \quad 1$, if facility at location $j \in J$ is open; 0 , otherwise

$M_{j}^{\beta} \quad \mathrm{CBM}$ of the distances travelled by facility $j \in J$ to serve demand points

\section{Primal Formulation}

$$
\begin{array}{cc}
\text { MODEL 1A: } & \\
\sum_{j \in J} x_{i j} \leq 1 & \\
\sum_{j \in J} y_{j} \leq p & \forall i \in I \\
\sum_{i \in I} w_{i} x_{i j} \leq U_{j} y_{j} & \\
x_{i j} \leq y_{j} x_{i j} & \forall j \in J \\
\sum_{i \in I} x_{i j} \geq y_{j} & \forall i \in I, j \in J \\
M_{j}^{\beta} \leq r_{j} & \forall j \in J
\end{array}
$$




$$
\begin{array}{rr}
x_{i j} \in\{0,1\} & \forall i \in I, j \in J \\
y_{j} \in\{0,1\} & \forall j \in J
\end{array}
$$

where, $\forall j \in J$, we define

$$
\begin{gathered}
W_{j}^{\beta}=\beta \sum_{i \in I} w_{i} x_{i j} \\
M_{j}^{\beta}=\operatorname{Max}\left\{\frac{1}{W_{j}^{\beta}} \sum_{i \in I} d_{i j} x_{i j} z_{i j}\right\} \\
\sum_{i \in I} z_{i j} \leq W_{j}^{\beta} \\
z_{i j} \leq w_{i} \\
z_{i j} \geq 0
\end{gathered} \quad \forall i \in I
$$

MODEL-1A represents the formulation of a capacitated maximum covering problem with equity incorporated using CBM constraints. The goal of the objective function in equation 3.2.1 is to maximize the total demand served by the open facilities. Constraint 3.2.2 ensures that each demand point is only served by one facility. Constraint 3.2.3 limits the number of facilities that could be open to p. Constraint 3.2.4 ensures that the total demand served by a facility is less than its capacity. Constraint 3.2.5 makes sure that demands point are only assigned to open facilities. Equation 3.2.5 is a redundant constraint, as it is implicitly incorporated within equation 3.2.4. However, the presence of equation 3.2.5 improves 
the linear relaxation and makes solving the model easier. Constraint 3.2.6 enforces that each open facility serves at least one demand point. Equations 3.2.8 and 3.2.9 define the binary variables in this model. As stated earlier in this section, constraint 3.2.7 enforces each facility's CBM to be lower than their coverage radius.

Equations 3.2.10-3.2.14 represent another optimization problem defined to calculate the CBM. The objective function in equation 3.2.11 gives the CBM for each facility $j \in J$. Equation 3.2.10 calculates the amount of demand served by facility j corresponding to $\beta$. Constraints 3.2.12 and 3.2.13 collectively help in finding the farthest $\beta$ share of demands. Equation 3.2.14 defines the variables of the objective function in equation 3.2.11.

\section{Dualizing CBM Formulation}

Consider the equation provided below,

$$
\frac{1}{W_{j}^{\beta}} \sum_{i \in I} d_{i j} x_{i j} z_{i j} \leq r_{j} \quad \forall j \in J
$$

If this equation is provided as a constraint to the maximizing objective in equation 3.2.1, it is incentivized to allocate weights $z_{i j}$ to demand points nearest to the facility, which naturally implies minimize the LHS.

However, the objective is to allocate weights to the farthest demand points served by the facility, so it is maximized in equation 3.2.11. Since the maximiza- 
tion in equation 3.2.7 and 3.2.11 conflicts with the global maximization in equation 3.2.1, dualization is used to swap-off and combine both objectives.

The CBM formulation, in equations 3.2.11-3.2.14, is dualized with respect to $z$ variables. The resultant dualized formulation for CBM is given as:

$$
\begin{array}{cc}
M_{j}^{\beta}=\frac{1}{W_{j}^{\beta}} \operatorname{Min}\left\{W_{j}^{\beta} \gamma_{j}+\sum_{i \in I} w_{i} \delta_{i j}\right\} & \\
\gamma_{j}+\delta_{i j} \geq d_{i j} x_{i j} & \forall i \in I \\
\delta_{i j} \geq 0 & \forall i \in I \\
\gamma_{j} \geq 0 &
\end{array}
$$

In equation 3.2.15, the parameter $W_{j}^{\beta}$ is independent of $z$ variables, so it is taken out of the minimization function. The variable $\gamma$ is the dual variable associated with constraint 3.2.12, and the variable $\delta$ is the dual variable associated with constraint 3.2.13. Now, after dualization, the updated formulation is given as:

$$
\begin{array}{rr}
\operatorname{Max} \sum_{i \in I} \sum_{j \in J} w_{i} x_{i j} & \\
(3.2 .2)-(3.2 .6) & \\
W_{j}^{\beta} \gamma_{j}+\sum_{i \in I} w_{i} \delta_{i j} \leq r_{j} W_{j}^{\beta} & \forall j \in J \\
\gamma_{j}+\delta_{i j} \geq d_{i j} x_{i j} & \forall i \in I, j \in J
\end{array}
$$




$$
\begin{aligned}
x_{i j}, y_{j} \in\{0,1\} & \forall i \in I, j \in J \\
\delta_{i j}, \gamma_{j} \geq 0 & \forall i \in I, j \in J
\end{aligned}
$$

Using equation 3.2.10, equation 3.2.19 can be expanded and re-written as equation 3.2.23.

$$
\beta \sum_{i \in I} w_{i} x_{i j} \gamma_{j}+\sum_{i \in I} w_{i} \delta_{i j} \leq \beta \sum_{i \in I} w_{i} x_{i j} r_{j} \quad \forall j \in J
$$

Consider the product of the two variables $x_{i j}$ and $\gamma_{j}$ as $\theta_{i j}$, i.e., $\theta_{i j}=x_{i j} \gamma_{j}$. This product of variables can be linearized as in Coelho (2013) by replacing constraint 3.2.23 with the following set of constraints. Here, $\mathrm{M}$ is a very large number, with a value higher than any variable in model.

$$
\begin{array}{cr}
\beta \sum_{i \in I} w_{i} \theta_{i j}+\sum_{i \in I} w_{i} \delta_{i j} \leq \beta \sum_{i \in I} w_{i} x_{i j} r_{j} & \forall j \in J \\
\theta_{i j} \leq M x_{i j} & \forall i \in I, j \in J \\
\theta_{i j} \leq \gamma_{j} & \forall i \in I, j \in J \\
\theta_{i j} \geq \gamma_{j}-\left(1-x_{i j}\right) M & \forall i \in I, j \in J \\
\theta_{i j} \geq 0 & \forall i \in I, j \in J
\end{array}
$$

Now, after performing dualization and adding above changes to MODEL-1A, 
the final formulation is given as following MODEL-1B:

MODEL 1B: $\quad \operatorname{Max} \sum_{i \in I} \sum_{j \in J} w_{i} x_{i j}$

s.t.

$$
\begin{array}{cr}
\sum_{j \in J} x_{i j} \leq 1 & \forall i \in I \\
\sum_{j \in J} y_{j} \leq p & \\
\sum_{i \in I} w_{i} x_{i j} \leq U_{j} y_{j} & \forall j \in J \\
x_{i j} \leq y_{j} & \forall i \in I, j \in J \\
\sum_{i \in I} x_{i j} \geq y_{j} & \forall j \in J \\
\beta \sum_{i \in I} w_{i} \theta_{i j}+\sum_{i \in I} w_{i} \delta_{i j} \leq \beta \sum_{i \in I} w_{i} x_{i j} r_{j} & \forall j \in J \\
\gamma_{j}+\delta_{i j} \geq d_{i j} x_{i j} & \forall i \in I, j \in J \\
\theta_{i j} \leq M x_{i j} & \forall i \in I, j \in J \\
\theta_{i j} \leq \gamma_{j} & \forall i \in I, j \in J \\
\theta_{i j} \geq \gamma_{j}-\left(1-x_{i j}\right) M & \forall i \in I, j \in J \\
\theta_{i j}, \delta_{i j}, \gamma_{j} \geq 0 & \forall i \in I, j \in J \\
x_{i j}, y_{j} \in\{0,1\} & \forall i \in I, j \in J
\end{array}
$$




\subsection{MCLP-CBM-II Model}

In this section, we provide another version of the MCLP model incorporated with CBM. In this model, instead of $\beta \%$ demands, CBM for a facility is defined as the average distance of the farthest $\beta$ number of demands served by it. So, here the parameter $\beta$ is a positive integer. Adopting the nomenclature and formulation of MODEL-1A, the following MODEL-2A with the new definition of CBM is developed.

While solving an MCLP, though rare, the number of demands served by a facility can be less than $\beta$. To calculate the average distances of demands, an exact number of demands served by a facility is necessary in such cases. A new variable $\alpha_{j}$ is introduced, whose value would be either number of facilities served

or $\beta$, whichever is lower. The following variables and parameters are added to the nomenclature of MODEL-1A.

Parameters

$\beta \quad$ Maximum number of points to be used for estimating CBM

Variables

$\alpha_{j} \quad$ Number of demand points actually considered for estimating CBM of facility $j \in J$ 


\section{Primal Formulation}

$$
\begin{array}{cc}
\text { MODEL 2A: } & \\
\sum_{j \in J} x_{i j} \leq 1 & \\
\sum_{j \in J} y_{j} \leq p & \\
\sum_{i \in I} w_{i} x_{i j} \leq U_{j} y_{j} w_{i} x_{i j} & \\
x_{i j} \leq y_{j} & \forall j \in J \\
M_{j}^{\beta} \leq r_{j} & \\
\alpha_{j} \leq \beta_{j} & \forall i \in I, j \in J \\
\sum_{i \in I} x_{i j} \geq \alpha_{j}+y_{j}-1 & \forall j \in J \\
x_{i j}, y_{j} \in\{0,1\} & \forall j \in J \\
\alpha_{j} \in N & \forall j \in J
\end{array}
$$

where, $\forall j \in J$, we define

$$
\begin{array}{cc}
M_{j}^{\beta}=\operatorname{Max}\left\{\frac{1}{\alpha_{j}} \sum_{i \in I} d_{i j} x_{i j} z_{i j}\right\} & \\
\sum_{i \in I} z_{i j} \leq \alpha_{j} & \forall i \in I \\
z_{i j} \leq 1 & \forall i \in I \\
z_{i j} \geq 0 & \forall i \in I
\end{array}
$$


MODEL-2A represents the formulation of a capacitated maximum covering problem with equity incorporated using CBM constraints. The objective function and constraints, in equations 3.3.1-3.3.5, have the same purpose as in MODEL$1 \mathrm{~A}$.

Constraint 3.3.7 ensures that $\alpha_{j}$ value is not more than $\beta_{j}$, the user-defined threshold. Next, if a facility $j \in J$ is open, $y_{j}=1$, which will make the constraint 3.3.8 appear as $\sum_{i \in I} x_{i j} \geq \alpha_{j}$. So, the $\alpha_{j}$ does not exceed the total number of demand points served by a facility if this total is lower than $\beta$. When a facility $j \in J$ is closed, all corresponding $x_{i j}$ are made 0 by constraint 3.3 .5 , and $\alpha_{j}$ has the possibility to assume 1 to make the model feasible. Equation 3.3.9 is defining the binary variables. Equation 3.3.10 defines the $\alpha_{j}$ variables to be positive integers or natural numbers.

Equations 3.3.11-3.3.14 are part of the maximization problem defined to estimate CBM. The objective function in equation 3.3.11 calculates the CBM of distances for each facility $j \in J$. Constraint 3.3.12 helps in finding the farthest $\alpha_{j}$

demand points for facility $j \in J$. Equations 3.3.13 and 3.3.14 define the upper and lower bounds of the variables in the objective function in equation 3.3.11.

\section{Dual CBM Formulation}

Similar to the model in previous section, the maximization in equation 3.3.11 conflicts with the global maximization in equation 3.3.1. So, the CBM formulation, in equations 3.3.11-3.3.14, is dualized with respect to $z$ variables. The 
resultant dualized formulation is as follows:

$$
\begin{array}{cc}
M_{j}^{\beta}=\frac{1}{\alpha_{j}} \operatorname{Min}\left\{\alpha_{j} \gamma_{j}+\sum_{i \in I} \delta_{i j}\right\} & \\
\gamma_{j}+\delta_{i j} \geq d_{i j} x_{i j} & \forall i \in I \\
\delta_{i j} \geq 0 & \forall i \in I \\
\gamma_{j} \geq 0 &
\end{array}
$$

In equation 3.3.15, the parameter $\alpha_{j}$ is independent of $\mathrm{z}$ variables, so it is outside the minimization function. The variable $\gamma$ is the dual variable associated with constraint 3.3.12, and the variable $\delta$ is the dual variable associated with constraint 3.3.13. Now, after dualization, the updated formulation is given as:

$$
\operatorname{Max} \sum_{i \in I} \sum_{j \in J} w_{i} x_{i j}
$$

$$
\begin{array}{rr}
\alpha_{j} \gamma_{j}+\sum_{i \in I} \delta_{i j} \leq r_{j} \alpha_{j} & \forall j \in J \\
\gamma_{j}+\delta_{i j} \geq d_{i j} x_{i j} & \forall i \in I, j \in J \\
x_{i j}, y_{j} \in\{0,1\} & \forall i \in I, j \in J \\
\alpha_{j} \in N & \forall j \in J \\
\delta_{i j}, \gamma_{j} \geq 0 & \forall i \in I, j \in J
\end{array}
$$


In equation 3.3.19, the product of integer variable $\alpha_{j}$ and continuous variable $\gamma_{j}$ rises non-linearity in the model, and this will be linearized in two steps. In first step, the integer variable $\alpha_{j}$ is converted into a series of binary variables. Next, the product of binary and continuous variables is linearized as in Coelho (2013).

With constraint 3.3.7, $\alpha_{j}$ would never exceed $\beta_{j}$. Now for each $\alpha_{j}$, consider a set $K_{j}$ such that $K_{j} \in\left\{1,2,3, \ldots, \beta_{j}\right\}$. Now introduce new binary variables $\phi_{j k}$, where $j \in J$ and $k \in K_{j}$.

The following equations 3.3.24 and 3.3.25 will ensure $\alpha_{j}$ to remain as a integer variable.

$$
\begin{array}{cc}
\alpha_{j}=\sum_{k \in K_{j}} k \phi_{j k} & \forall j \in J \\
\sum_{k \in K_{j}} \phi_{j k}=1 & \forall j \in J
\end{array}
$$

With equation 3.3.24, equation 3.3.19 can be re-written as equation 3.3.26.

$$
\sum_{k \in K_{j}} k \phi_{j k} \gamma_{j}+\sum_{i \in I} \delta_{i j} \leq r_{j} \sum_{k \in K_{j}} k \phi_{j k} \quad \forall j \in J
$$

Now consider the product of $\phi_{j k}$ and $\gamma_{j}$ as $\epsilon_{j k}$, i.e., $\phi_{j k} \gamma_{j}=\epsilon_{j k}$. Now equation 3.3.26 can be replaced with the following set of equations for linearization.

$$
\sum_{k \in K_{j}} k \epsilon_{j k}+\sum_{i \in I} \delta_{i j} \leq r_{j} \sum_{k \in K_{j}} k \phi_{j k} \quad \forall j \in J
$$




$$
\begin{aligned}
\epsilon_{j k} \leq M \phi_{j k} & \forall j \in J, k \in K_{j} \\
\epsilon_{j k} \leq \gamma_{j} & \forall j \in J, k \in K_{j} \\
\epsilon_{j k} \geq \gamma_{j}-\left(1-\phi_{j k}\right) M & \forall j \in J, k \in K_{j} \\
\epsilon_{j k} \geq 0 & \forall j \in J, k \in K_{j}
\end{aligned}
$$

Now after performing the dualization, linearization, and updating the above changes to Model-2A, the final formulation is given as following MODEL-2B:

MODEL 2B: $\quad \operatorname{Max} \sum_{i \in I} \sum_{j \in J} w_{i} x_{i j}$

s.t.

$$
\begin{array}{cc}
\sum_{j \in J} x_{i j} \leq 1 & \forall i \in I \\
\sum_{j \in J} y_{j} \leq p & \\
\sum_{i \in I} w_{i} x_{i j} \leq U_{j} y_{j} & \forall j \in J \\
\sum_{i \in I} x_{i j} \geq \sum_{k \in K_{j}} x_{i j} \leq y_{j}+y_{j}-1 & \forall j \in J \\
\sum_{k \in K_{j}} k \phi_{j k} \leq \beta_{j} & \forall j \in J \\
\sum_{k \in K_{j}} \phi_{j k}=1 & \forall j \in J \\
\gamma_{j}+\delta_{i j} \geq d_{i j} x_{i j} & \forall j \in J
\end{array}
$$




$$
\begin{array}{rr}
\sum_{k \in K_{j}} k \epsilon_{j k}+\sum_{i \in I} \delta_{i j} \leq r_{j} \sum_{k \in K_{j}} k \phi_{j k} & \forall j \in J \\
\epsilon_{j k} \leq M \phi_{j k} & \forall j \in J, k \in K_{j} \\
\epsilon_{j k} \leq \gamma_{j} & \forall j \in J, k \in K_{j} \\
\epsilon_{j k} \geq \gamma_{j}-\left(1-\phi_{j k}\right) M & \forall j \in J, k \in K_{j} \\
x_{i j}, y_{j}, \phi_{j k} \in\{0,1\} & \forall i \in I, j \in J, k \in K_{j} \\
\delta_{i j}, \gamma_{j}, \epsilon_{j k} \geq 0 & \forall i \in I, j \in J, k \in K_{j} \\
K_{j}=\left\{1,2,3, \ldots, \beta_{j}\right\} & \forall j \in J
\end{array}
$$




\section{EMS Location Problem Application}

This chapter focuses on incorporating CBM into an EMS facility location

problem. First, an EMS location problem called the maximum expected survival location problem (MEXSLP) is explained. Next, a new model called MEXSLPCBM-I is proposed and developed by integrating the concept of CBM into the classical MEXSLP as a constraint. Lastly, an alternative way of incorporating CBM is defined, and another variant of the MEXSLP called MEXSLP-CBM-II is developed.

\subsection{MEXSLP Problem Description}

Daskin (1983) introduced the maximum expected covering location problem (MEXCLP), which considers the busy probabilities of facilities and aims to allocate the available EMS units across the potential facility locations in the system. Erkut et al. (2008) introduced a similar model called the maximum expected survival location problem (MEXSLP). The parameters in MEXSLP consider the probability of survival of patients while allocating EMS units to potential locations.

In MEXCLP, a demand point is covered if there are EMS units within the 
response time threshold. However, if no units are present in the said threshold, the next closest unit will be sent to the demand location. In MEXSLP, if the closest EMS unit is not available, the next closest is chosen for service, and this is achieved by maximizing the overall expected probability of survival in the system.

The MEXSLP model provided in Erkut et al. (2008) is nonlinear. Jagtenberg and Mason (2020) presented a linear version of the same, which again turns nonlinear after they incorporate equity into their model. Though MEXSLP is more realistic than other models, it does not necessarily provide an equitable solution for urban, suburban and rural localities. Since the population or fraction of the population is used as weights for the objective function in MEXSLP, their solutions allocate most EMS units nearer to densely populated or urban areas. This could result in longer travel times for people in suburban and rural areas.

The CBM measure is used in this study to reduce this disparity among densely and sparsely populated localities. CBM measures the average value of service provided for the $\beta \%$ of demand that is worst off. Filippi et al. (2019a) uses this measure in a bi-objective model to equitably locate facilities. In our model, CBM is used as a constraint such that the CBM of travel times of each demand point does not exceed the response time threshold.

There are several functions to estimate the probability of survival in the literature. Next, we present two such survival functions that were highlighted in Jagtenberg and Mason (2020). The first function was introduced in Valenzuela 
et al. (1997); it uses the time from collapse to performing CPR $\left(t_{C P R}\right)$ and the time from collapse to defibrillation $\left(t_{\text {defib }}\right)$ as variables. This function is given by:

$$
f(t)=\left(1+e^{-0.260+0.106 t_{C P R}+0.139 t_{d e f i b}}\right)^{-1}
$$

The second function was introduced in De Maio et al. (2003), which uses the EMS unit response time (t) as a variable to estimate the probability of survival and it is given by:

$$
f(t)=\left(1+e^{0.679+0.262 t}\right)^{-1}
$$

Functions defined in equations 4.1 .1 and 4.1.2, are monotonically decreasing with respect to travel time. Though we mentioned only two such functions, the models stated in this chapter are valid for any monotonically decreasing survival functions.

Our study assumes that all the EMS units in the system are ALS units, and all the calls received are life-threatening, requiring immediate treatment. It is also assumed that all the patients are first treated by the ALS units on-site, next they are transported to the nearest hospitals or trauma centers, and lastly, these ALS 
EMS units go back to their initial assigned stations. So, relocation or re-routing of EMS units are not considered.

\subsection{MEXSLP-CBM-I Model}

This model considers a set $I$ containing all demand points and another set $J$ with all potential locations to set up facilities with EMS units. Each facility location can have multiple EMS units, and there is only $p$ number of units in the entire system. In this model, a unique priority number is assigned to all $p$ units concerning each demand point. Units closer to the demand location get higher priority, i.e., lower priority number. Every demand points $i \in I$ is assumed to have atleast one potential facility location whose travel time is less than the response time threshold.

The EMS unit closest to the demand point might not be available all the time, so a busy probability is considered for each unit. This busy probability is assumed to be the same for all facilities. Parameter $q$ represents the average fraction of time a unit is busy or unavailable for service.

This model aims to maximize the expected probability of survival for this system while allocating the available EMS units optimally and equitably. The MEXSLP part of the formulation takes care of placing the units optimally. The CBM measure is used to achieve equity. 
We consider the equation 4.1.2 for calculating probability of survival, which is a monotonically decreasing function of time. So, maximizing to find facilities with the highest probability of survival would give the same output as minimizing to find facilities with the lowest travel times. The $\beta$ facilities with the highest survival probability are the ones with the lowest travel times. The CBM for each demand point is measured as the average time needed by the nearest $\beta \%$ EMS units to arrive at the location. This CBM of each location is kept lower than the response time threshold $T$.

\section{Nomenclature}

Sets

I Set of all demand points

$J \quad$ Set of all potential facility locations

Indices

$$
\begin{aligned}
& i \in I \\
& j \in J
\end{aligned}
$$

Parameters 
$w_{i} \quad$ Demand of point $i \in I$

$t_{i j} \quad$ Estimated travel time from facility $j \in J$ to demand point $i \in I$

$p \quad$ Maximum number of EMS units available

$q \quad$ Vehicle busy fraction, i.e., the average fraction of time an EMS unit is busy

$s_{i j} \quad$ Probability of survival when an EMS unit goes from facility $j \in J$ to demand $i \in I$

T Response Time Threshold

$\beta \quad$ Percentile of EMS units considered for calculating CBM

Variables

$x_{j} \quad$ Number of EMS units placed at location $j \in J$

$y_{i j k} \quad 1$, if $k^{t h}$ closest EMS unit for demand $i \in I$ is located at facility $j \in J ; 0$, otherwise

$M_{i}^{\beta} \quad \mathrm{CBM}$ of travel times of farthest $\beta$-percentile EMS units for demand $i \in I$

\section{Primal Formulation}

$$
\begin{aligned}
\text { MODEL 3A: } \quad & \operatorname{Max} \sum_{i \in I} \sum_{j \in J} \sum_{k=1}^{p} w_{i}(1-q) q^{k-1} s_{i j} y_{i j k} \\
& \forall i \in I, k \in\{1,2, \ldots, p\} \\
\sum_{j \in J} y_{i j k}=1 & \forall
\end{aligned}
$$




$$
\begin{array}{cr}
\sum_{k=1}^{p} y_{i j k}=x_{j} & \forall i \in I, j \in J \\
\sum_{j \in J} x_{j} \leq p & \\
M_{i}^{\beta} \leq T & \forall i \in I \\
x_{j} \in Z^{+} \cup\{0\} & \forall j \in J \\
y_{i j k} \in\{0,1\} & \forall i \in I, j \in J, k \in\{1,2, \ldots, p\}
\end{array}
$$

where, $\forall i \in I$, we define

$$
\begin{gathered}
W_{i}^{\beta}=\beta \sum_{j \in J} \sum_{k=1}^{p} y_{i j k} \\
M_{i}^{\beta}=\operatorname{Min}\left\{\frac{1}{W_{i}^{\beta}} \sum_{j \in J} \sum_{k=1}^{p} t_{i j} y_{i j k} z_{i j k}\right\} \\
\sum_{j \in J} \sum_{k=1}^{p} z_{i j k} \geq W_{i}^{\beta} \\
z_{i j k} \in\{0,1\} \quad \forall j \in J, k \in\{1,2, \ldots, p\}
\end{gathered}
$$

The objective function in equation 4.2.1 aims to maximize the expected survival probability of the entire system. $\sum_{j \in J} \sum_{k=1}^{p}(1-q) q^{k-1} s_{i j} y_{i j k}$ part of the objective function calculates the expected survival probability for each demand location $i \in I$. Constraint 4.2.17 and 4.2.18 ensures that each demand location has only one EMS unit which is $k^{t h}$ closest to it. Constraint 4.2 .17 also ensures that each unit gets a unique priority number specific to each demand location. 
Constraint 4.2.19 limits the total number of units allocated in the system to be p. Constraint 4.2.5 enforces the CBM measure of each demand location to be lower than the response time threshold. Equations 4.2.6 and 4.2.7 define the model variables.

Equations 4.2.8-4.2.11 represent the formulation of CBM optimization problem. Objective function in equation 4.2.9 gives the mean travel time of $\beta \%$ units nearest to demand $i$. Constraint 4.2.10 enforces the number of units in CBM calculation to be $\beta \%$ for each demand $i$. Equation 4.2 .11 defines the binary variables in CBM problem. Constraints 4.2.9 and 4.2.10 together find the $\beta \%$ units nearest to demand point $i$. From constraint 4.2.17, $\sum_{j \in J} \sum_{k=1}^{p} y_{i j k}=p$, so $W_{i}^{\beta}$ becomes $p$.

The objective function in equation 4.2.1 is naturally incentivized to minimize $y_{i j k}$ to reduce travel times so that the probability of survival is maximized. Equation 4.2.9 is also incentivized to allocate weights to minimize the travel times to find the shortest routes. Since both the objectives are in harmony, the inner minimization can be removed, and the combined formulation is as follows:

$$
\begin{gathered}
\operatorname{Max} \sum_{i \in I} \sum_{j \in J} \sum_{k=1}^{p} w_{i}(1-q) q^{k-1} s_{i j} y_{i j k} \\
(4.2 .17)-(4.2 .19) \\
\frac{1}{\beta p}\left\{\sum_{j \in J} \sum_{k=1}^{p} t_{i j} y_{i j k} z_{i j k}\right\} \leq T \\
\end{gathered} \quad \forall i \in I
$$




$$
\begin{array}{cr}
\sum_{j \in J} \sum_{k=1}^{p} z_{i j k} \geq \beta p & \forall i \in I \\
x_{j} \in Z^{+} \cup\{0\} & \forall j \in J \\
y_{i j k}, z_{i j k} \in\{0,1\} & \forall i \in I, j \in J, k \in\{1,2, \ldots, p\}
\end{array}
$$

By definition of variable $y_{i j k}$ tells if facility $j \in J$ has the $k^{\text {th }}$ nearest unit for demand point $i \in I$. For any demand $i$, the variables $y_{i j k}$ with $k \in\{1,2, \ldots,\lceil p \beta\rceil\}$ provide the nearest $\beta \%$ units. So, equations 4.2 .12 and 4.2 .13 can be replaced with equation 4.2.20, and all the $z_{i j k}$ variables can be eliminated. After all the above stated changes, the final combined formulation is given as following MODEL3B.

$$
\text { MODEL 3B: } \quad \operatorname{Max} \sum_{i \in I} \sum_{j \in J} \sum_{k=1}^{p} w_{i}(1-q) q^{k-1} s_{i j} y_{i j k}
$$

s.t.

$$
\begin{aligned}
& \sum_{j \in J} y_{i j k}=1 \quad \forall i \in I, k \in\{1,2, \ldots, p\} \\
& \sum_{k=1}^{p} y_{i j k}=x_{j} \quad \forall i \in I, j \in J \\
& \sum_{j \in J} x_{j} \leq p \\
& \frac{1}{\lceil\beta p\rceil} \sum_{j \in J} \sum_{k=1}^{\lceil\beta\rceil} t_{i j} y_{i j k} \leq T \\
& \forall i \in I
\end{aligned}
$$




$$
\begin{array}{rr}
x_{j} \in Z^{+} \cup\{0\} & \forall j \in J \\
y_{i j k} \in\{0,1\} \quad \forall i \in I, j \in J, k \in\{1,2, \ldots, p\}
\end{array}
$$

\subsection{MEXSLP-CBM-II Model}

In this section, we propose another version of the MEXSLP model incorporating CBM. Similar to MEXSLP-CBM-I, this model also aims to maximize the expected probability of survival for the system while allocating the available EMS units optimally and equitable. However, the CBM measure used to achieve equity is defined in different way. The CBM is defined as the mean of the longest $\beta \%$ travel times along the routes with same priority. Here, we consider the routes with first, second and third priorities and the CBM specific to each priority is kept lower than the response time threshold $\mathrm{T}$.

Adopting the nomenclature and formulation of MODEL-3A, the following MODEL-4A with the new definition of CBM is developed. The new CBM variable is defined as follows:

Variables

$M_{k}^{\beta} \quad$ CBM of travel times of farthest $\beta$-percentile EMS units having $k^{\text {th }}$ priority 


\section{Primal Formulation}

$$
\text { MODEL 4A: } \quad \operatorname{Max} \sum_{i \in I} \sum_{j \in J} \sum_{k=1}^{p} w_{i}(1-q) q^{k-1} s_{i j} y_{i j k}
$$

s.t.

$$
\begin{aligned}
& \sum_{j \in J} y_{i j k}=1 \quad \forall i \in I, k \in\{1,2, \ldots, p\} \\
& \sum_{k=1}^{p} y_{i j k}=x_{j} \quad \forall i \in I, j \in J \\
& \sum_{j \in J} x_{j} \leq p \\
& M_{k}^{\beta} \leq T \\
& \forall k \in\{1,2,3\} \\
& x_{j} \in Z^{+} \cup\{0\} \\
& \forall j \in J \\
& y_{i j k} \in\{0,1\} \quad \forall i \in I, j \in J, k \in\{1,2, \ldots, p\}
\end{aligned}
$$

where, $\forall k \in\{1,2,3\}$, we define

$$
\begin{gathered}
W_{k}^{\beta}=\beta \sum_{i \in I} \sum_{j \in J} y_{i j k} \\
M_{k}^{\beta}=\operatorname{Max}\left\{\frac{1}{W_{k}^{\beta}} \sum_{i \in I} \sum_{j \in J} t_{i j} y_{i j k} z_{i j k}\right\} \\
\sum_{i \in I} \sum_{j \in J} z_{i j k} \leq W_{k}^{\beta} \\
z_{i j k} \leq 1 \quad \forall i \in I, j \in J
\end{gathered}
$$




$$
z_{i j k} \geq 0 \quad \forall i \in I, j \in J
$$

MODEL-4A represents the formulation of a maximum expected survival location problem incorporated with CBM with respect first three priority EMS units, to provide equity in service for patients. Equations 4.3.1-4.3.7 define the same objective and constraints on this model as in MODEL-3A. Though constraint 4.3.5 is similar to constraint 4.2.5, what it enforces is different. Constraint 4.3.5 enforces the CBM for top three priority EMS units to be lower than the response time threshold.

Equations 4.3.8-4.3.12 represent the formulation of CBM optimization problem. The objective function in equation 4.3.9 gives the CBM of routes with given priority $k$. Equation 4.3 .8 calculates the $\beta \%$ of routes in system with $k^{\text {th }}$ priority. Constraints 4.3.10 and 4.3.11 together find the longest $\beta \%$ routes with $k^{\text {th }}$ priority. Equation 4.3.12 defines the variables in CBM optimization problem.

\section{Dualizing CBM Formulation}

Consider the equation provided below,

$$
\frac{1}{W_{k}^{\beta}} \sum_{i \in I} \sum_{j \in J} t_{i j} y_{i j k} z_{i j k} \leq T \quad \forall k \in\{1,2,3\}
$$

If this equation is provided as a constraint to the maximizing objective in 
equation 4.3.1, it is incentivized to allocate weights $z_{i j k}$ to shorter routes, which naturally implies the LHS to minimize.

However, the objective is to allocate weights to the longest routes, so it is maximized in equation 4.3.9. Since the maximization in equation 4.3.5 and 4.3.9 conflicts with the global maximization in equation 4.3.1, dualization is used to swap-off and combine both objectives.

The CBM formulation, in equations 4.3.9-4.3.12, is dualized with respect to $z$ variables. The resultant dualized formulation for CBM is given as:

$$
\begin{aligned}
M_{k}^{\beta}=\frac{1}{W_{k}^{\beta}} \operatorname{Min}\left\{W_{k}^{\beta} \gamma_{k}+\sum_{i \in I} \sum_{j \in J} \delta_{i j k}\right\} & \\
\gamma_{k}+\delta_{i j k} \geq t_{i j} y_{i j k} & \forall i \in I, j \in J \\
\delta_{i j k} \geq 0 & \forall i \in I, j \in J \\
\gamma_{k} \geq 0 &
\end{aligned}
$$

In equation 4.3 .13 , the parameter $W_{k}^{\beta}$ is independent of $z$ variables, so it is out of the minimization function. The variable $\gamma$ is the dual variable associated with constraint 4.3.11, and the variable $\delta$ is the dual variable associated with constraint 4.3.11. From constraint 4.3.18, $\sum_{i \in I} \sum_{j \in J} y_{i j k}=|I|$, so $W_{k}^{\beta}$ becomes $\beta|I|$. After dualization and making the stated changes, the combined final formulation 
is given as following MODEL-4B.

$$
\text { MODEL 4B: } \quad \operatorname{Max} \sum_{i \in I} \sum_{j \in J} \sum_{k=1}^{p} w_{i}(1-q) q^{k-1} s_{i j} y_{i j k}
$$

s.t.

$$
\begin{array}{rr}
\sum_{j \in J} y_{i j k}=1 & \forall i \in I, k \in\{1,2, \ldots, p\} \\
\sum_{k=1}^{p} y_{i j k}=x_{j} & \forall i \in I, j \in J \\
\sum_{j \in J} x_{j} \leq p & \\
\beta|I| \gamma_{k}+\sum_{i \in I} \sum_{j \in J} \delta_{i j k} \leq \beta|I| T & \forall k \in\{1,2,3\} \\
\gamma_{k}+\delta_{i j k} \geq t_{i j} y_{i j k} & \forall i \in I, j \in J, k \in\{1,2,3\} \\
\delta_{i j k}, \gamma_{k} \geq 0 & \forall i \in I, j \in J, k \in\{1,2,3\} \\
x_{j} \in Z^{+} \cup\{0\} & \forall j \in J \\
y_{i j k} \in\{0,1\} \quad \forall i \in I, j \in J, k \in\{1,2, \ldots, p\}
\end{array}
$$




\section{Analysis}

This chapter provides analysis on the CBM incorporated MCLP and MEXSLP models developed in chapters 3 and 4 . Here we will see that the implementation of CBM will improve EMS units' spread or uniformity in the concentration of EMS units. Also, the implementation of CBM in MCLP will help in creating a flexible coverage radius and increase total coverage in the whole service area.

The primary data used for testing is the Portland Metropolitan Region

dataset presented in Chauhan et al. (2019). Besides, the Portland Metropolitan dataset, a p-median model test case from Osman and Christofides (1994) available on J. E. Beasley's OR-library, is also used for MCLP models testing. All the computational runs are performed on a Windows 10 desktop having Intel Core i7-7700 CPU 3.6 GHz, 4 Cores, 8 Logical Processors, and 32 GB of RAM. Gurobi Optimizer 9.0 is used as the MILP solver in this study [Gurobi Optimization (2020)].

\subsection{MCLP-CBM Models Analysis}

\subsubsection{Analysis on Portland Metropolitan Data}

The Portland Metropolitan dataset consists of 122 demand points and 104 potential facility locations. Each demand point is the centroid of a zip code area, 
and each facility location is the location of a community center in the Portland Metropolitan Area. Here, the demand points and facility locations do not overlap with each other. Figure 5.1 shows the locations of all demand and potential facilities considered for this study.

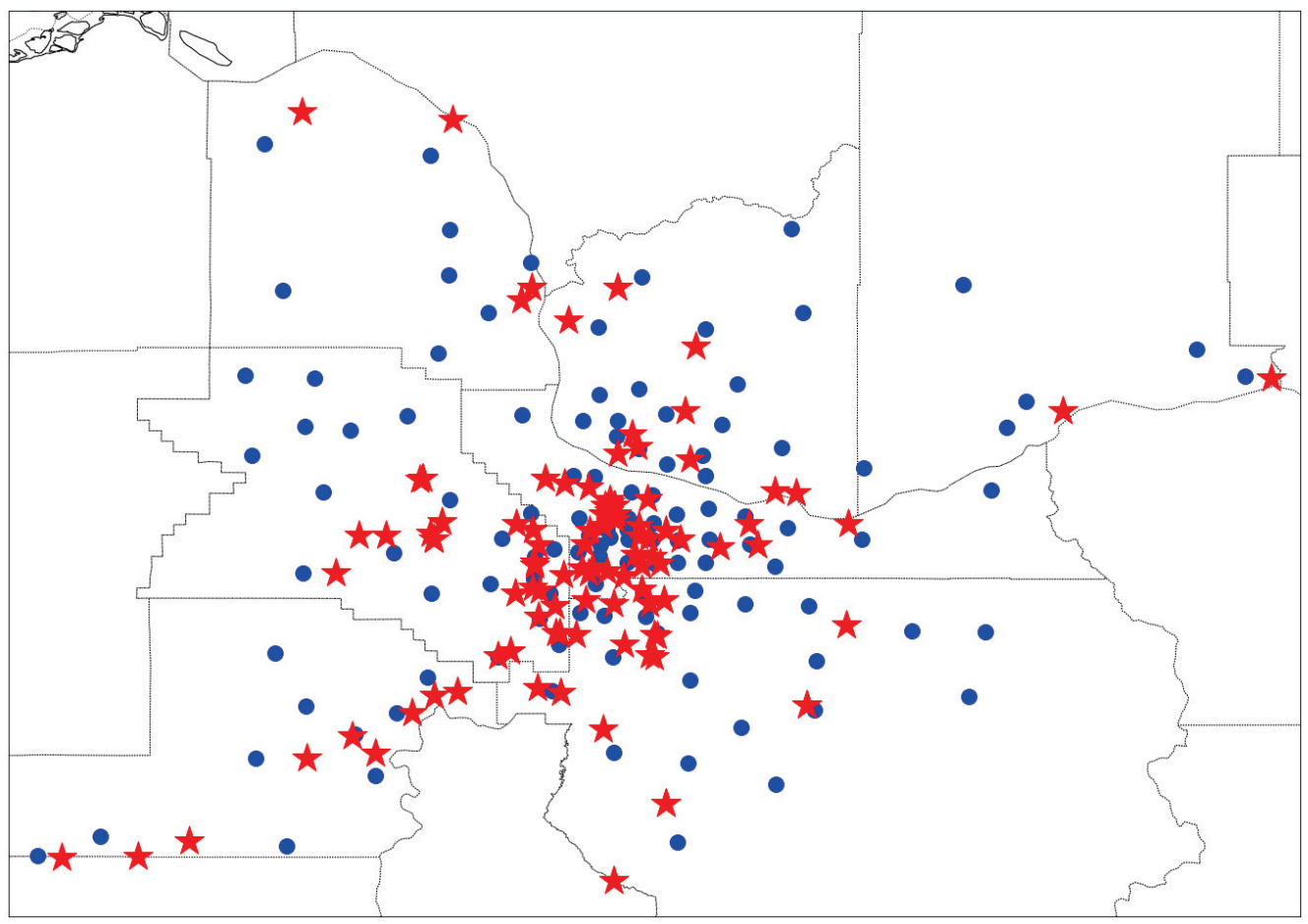

Figure 5.1: Demand points and potential facilities in the Portland Metropolitan Region.

From the American Community Survey (2020), the number of Hispanic and Non-Hispanic people below the poverty line is estimated, and this population data is used for demands $w_{i}$ in this study (refer Appendix-A). The capacity of each facility $U$ is calculated as it is suggested in Pirkul and Schilling (1989), using the below equation: 


$$
U=\frac{\sum_{i \in I} w_{i}}{0.8 p}
$$

The above equation ensures all open facilities' combined capacity to be $25 \%$ higher than the total demand to be served. As in Chauhan et al. (2019), the time required to travel the euclidian distance from facilities to demand sites is used as distance $d_{i j}$ in this study. The coverage radius $r_{j}$ is assumed to be the $20 t h$ percentile of all the distances in $d_{i j}$ matrix.

In MCLP models, the objective function is the total demand covered by all facilities in the service region. Table 5.1 provides the coverage and Gurobi gap values from three models: MCLP, MCLP-CBM-I, and MCLP-CBM-II models. All three MCLP models were tested with several combinations of $p$ and $\beta$. The number of facilities to open $p$ varying from 1 to 25 in multiples of 5 . The $\beta$ parameter used for calculating CBM to be 0.1\%, 10\%, 20\%, 30\%, and 40\% for MCLP-CBM-I model, and it to be 1, 2, 3, 5, and 10 for MCLP-CBM-II model.

For the analysis in table 5.1, the Gurobi solver was given a time limit of two hours. In two hours, the gap in Gurobi solutions of MCLP-CBM-I is very high, stating that the solution has not converged. In comparison, the MCLP-CBM-II model has much lower gaps, showing convergence in solution.

Besides convergence, as expected table 5.1 shows that coverage and $\beta$ are 
Table 5.1: MCLP Models - Objective and Gurobi Gap using PDX Dataset.

\begin{tabular}{|c|c|c|c|c|c|c|c|}
\hline \multirow[b]{2}{*}{$\mathrm{p}$} & \multirow{2}{*}{$\begin{array}{c}\text { MCLP } \\
\text { Coverage }(\%)\end{array}$} & \multirow{2}{*}{$\beta(\%)$} & \multicolumn{2}{|c|}{ MCLP CBM-I } & \multirow{2}{*}{$\beta(\#)$} & \multicolumn{2}{|c|}{ MCLP CBM-II } \\
\hline & & & Coverage $(\%)$ & $\operatorname{Gap}(\%)$ & & Coverage $(\%)$ & $\operatorname{Gap}(\%)$ \\
\hline \multirow{5}{*}{1} & \multirow{5}{*}{63.87} & 0.1 & 63.87 & 46.23 & 1 & 63.87 & 0 \\
\hline & & 10 & 65.35 & 53.01 & 2 & 63.87 & 0 \\
\hline & & 20 & 67.39 & 48.38 & 3 & 64.01 & 0 \\
\hline & & 30 & 70.78 & 25.53 & 5 & 68.44 & 0 \\
\hline & & 40 & 74.79 & 22.35 & 10 & 71.07 & 0 \\
\hline \multirow{5}{*}{5} & \multirow{5}{*}{91.66} & 0.1 & 88.31 & 13.24 & 1 & 91.66 & 0 \\
\hline & & 10 & 88.08 & 13.53 & 2 & 92.26 & 3.98 \\
\hline & & 20 & 89.55 & 11.67 & 3 & 91.84 & 8.05 \\
\hline & & 30 & 91.50 & 9.28 & 5 & 93.74 & 6.68 \\
\hline & & 40 & 98.01 & 2.03 & 10 & 96.00 & 4.17 \\
\hline \multirow{5}{*}{10} & \multirow{5}{*}{96.84} & 0.1 & 94.83 & 5.46 & 1 & 96.84 & 0.01 \\
\hline & & 10 & 94.46 & 5.86 & 2 & 96.84 & 2.04 \\
\hline & & 20 & 97.52 & 2.54 & 3 & 96.89 & 3.21 \\
\hline & & 30 & 97.97 & 2.07 & 5 & 98.71 & 1.31 \\
\hline & & 40 & 98.04 & 2 & 10 & 99.99 & 0.01 \\
\hline \multirow{5}{*}{15} & \multirow{5}{*}{98.12} & 0.1 & 96.57 & 3.55 & 1 & 98.12 & 0 \\
\hline & & 10 & 96.63 & 3.49 & 2 & 98.22 & 1.14 \\
\hline & & 20 & 98.77 & 1.25 & 3 & 98.51 & 1.52 \\
\hline & & 30 & 97.08 & 3 & 5 & 99.59 & 0.41 \\
\hline & & 40 & 99.36 & 0.65 & 10 & 100.00 & 0 \\
\hline \multirow{5}{*}{20} & \multirow{5}{*}{92.49} & 0.1 & 91.76 & 1.79 & 1 & 92.49 & 0 \\
\hline & & 10 & 91.63 & 1.94 & 2 & 92.70 & 0.61 \\
\hline & & 20 & 91.50 & 2.08 & 3 & 92.71 & 0.75 \\
\hline & & 30 & 92.76 & 0.69 & 5 & 93.41 & 0 \\
\hline & & 40 & 93.41 & 0 & 10 & 93.41 & 0 \\
\hline \multirow{5}{*}{25} & \multirow{5}{*}{92.77} & 0.1 & 92.46 & 1.02 & 1 & 92.77 & 0 \\
\hline & & 10 & 91.16 & 2.47 & 2 & 93.24 & 0.02 \\
\hline & & 20 & 92.82 & 0.63 & 3 & 93.26 & 0.16 \\
\hline & & 30 & 93.07 & 0.36 & 5 & 93.40 & 0.01 \\
\hline & & 40 & 93.41 & 0 & 10 & 93.41 & 0 \\
\hline
\end{tabular}


directly proportional. Higher the $\beta$, the higher the total coverage. Incorporating CBM in the MCLP loosens the constraint on coverage radius and allows the facilities with excess capacity to serve demands lying outside the coverage radius. As the $\beta$ increases, the facility's reach increases, allowing the model to increase its coverage. When $\beta$ gets lower, CBM tightens the constraint, and the MCLP-CBM models work similarly to a regular MCLP. When $\beta$ is $0.1 \%$ in MCLP-CBM-I or 1 in MCLP-CBM-II, the coverage provided by MCLP and MCLP-CBM models are the same. This validates our hypothesis that either of the MCLP-CBM models working similar to a regular MCLP when $\beta$ is low.

Both the MCLP-CBM models provided coverage close to each other and better than a regular MCLP. Though the coverage has improved, the gap in Gurobi solutions is in MCLP-CBM-I is way too high. Next, we will test the MCLP-CBM models using two standard test cases from Osman and Christofides (1994).

\subsubsection{Analysis on Standard Test Dataset}

The p-median dataset used in Osman and Christofides (1994) contains several test cases for maximum covering problems, out of which two were considered for this study. First, having 50 sites acting both as a potential facility and a demand point. Second has 100 sites which also act both as a potential facility location and a demand point. Model parameters such as $d_{i j}, p, U_{j}$ and $w_{i}$ are extracted from the dataset (refer Appendix-B). The coverage radius $r_{j}$ is assumed to be the 5 th 
percentile of distances in $d_{i j}$ matrix.

Table 5.2: MCLP Models - Objective and Gurobi Gap using P-Median Dataset.

\begin{tabular}{|c|c|c|c|c|c|c|c|}
\hline \multirow[b]{2}{*}{$\mathrm{p}$} & \multirow{2}{*}{$\begin{array}{c}\text { MCLP } \\
\text { Coverage }(\%)\end{array}$} & \multirow{2}{*}{$\beta(\%)$} & \multicolumn{2}{|c|}{ MCLP CBM-I } & \multirow{2}{*}{$\beta(\#)$} & \multicolumn{2}{|c|}{ MCLP CBM-II } \\
\hline & & & Coverage $(\%)$ & $\operatorname{Gap}(\%)$ & & Coverage $(\%)$ & $\operatorname{Gap}(\%)$ \\
\hline \multirow{3}{*}{5} & \multirow{3}{*}{48.37} & 1 & 48.37 & 0 & 1 & 48.37 & 0 \\
\hline & & 30 & 48.98 & 104.17 & 2 & 50.41 & 0 \\
\hline & & 60 & 57.55 & 73.76 & 5 & 69.80 & 0 \\
\hline \multirow{3}{*}{10} & \multirow{3}{*}{64.21} & 1 & 60.37 & 65.64 & 1 & 64.21 & 0 \\
\hline & & 30 & 68.93 & 45.08 & 2 & 73.75 & 11.47 \\
\hline & & 60 & 78.96 & 26.65 & 5 & 85.64 & 16.76 \\
\hline
\end{tabular}

Table 5.2 shows provides an analysis that is similar to table 5.1 using the two standard test cases taken from Osman and Christofides (1994). The three MCLP models were tested with $\beta$ value for calculating CBM to be $0.1 \%, 30 \%$ and $60 \%$ for MCLP-CBM-I model, and it to be 1, 2, and 5 for MCLP-CBM-II model.

Like previous analysis, the time limit for the Gurobi solver is set to be two hours. All the insights obtained from table 5.1 can also be observed in table 5.2. These insights include the coverage being proportional to $\beta$, MCLP-CBM models behaving similar to a regular MCLP with low $\beta$, and huge gap in Gurobi solutions.

The non-convergence or large gaps in Gurobi solutions make them unreliable. Especially for the MCLP-CBM-I model, where the gaps are as high as 104\%. To overcome non-convergence and reduce the solving time, a greedy heuristic of proposed to solve both the MCLP-CBM models in the next section. 


\subsection{Greedy Heuristic and Application}

\subsubsection{Heuristic Methodology}

The greedy heuristic works as follows:

i. Create two running arrays $I$ and $J$ with all demand points and potential facility locations.

ii. Create a weight array. For each unopened facility location, the weight is defined as the sum of all $w_{i}$ whose value is less than facility's capacity and located with in its coverage radius $r_{j}$.

iii. Find and open the facility with the highest weight.

iv. Now solve the MCLP-CBM model with the single newly opened facility and all unserved demand points, using Gurobi.

v. After solving the model with single facility, remove the opened facility and demand points assigned to it from arrays $I$ and $J$, respectively.

vi. Using the shortened arrays $I$ and $J$, again follow the procedure in steps (ii) to $(\mathrm{v})$.

vii. Keep repeating this cycle until required number of facilities are found and opened. 


\subsubsection{Heuristic Performance Analysis}

This greedy heuristic was tested with both the datasets discussed earlier. Their results obtained after solving with heuristic are provided in tables 5.3 and 5.4. These results include total coverage, solving time, and difference from Gurobi's solution. Observing these results, the heuristic seems to provide reliable results. To get the coverage provided Gurobi in two hours, the greedy heuristic only needs few seconds to minutes.

To understand the heuristic's performance, table 5.5 is prepared. Table 5.5 has the maximum, minimum, and average values of solving time and solution difference from Gurobi for both the datasets. In the best scenario, the greedy heuristic has solved the MCLP-CBM model under a second and obtain coverage equivalent to Gurobi. In the worst scenario, this heuristic solved the model in about two minutes and with coverage 10\% lower than Gurobi. Overall, the heuristic can be expected to solve the model in $10 \mathrm{sec}$ to obtain the same coverage as obtained using Gurobi for two hours. 


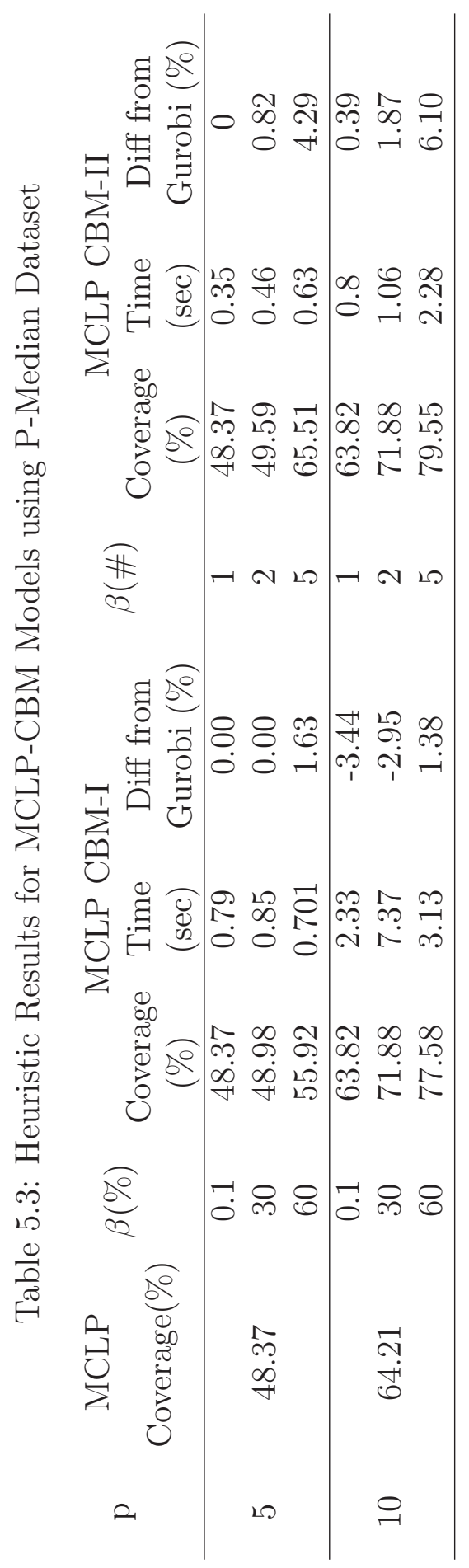




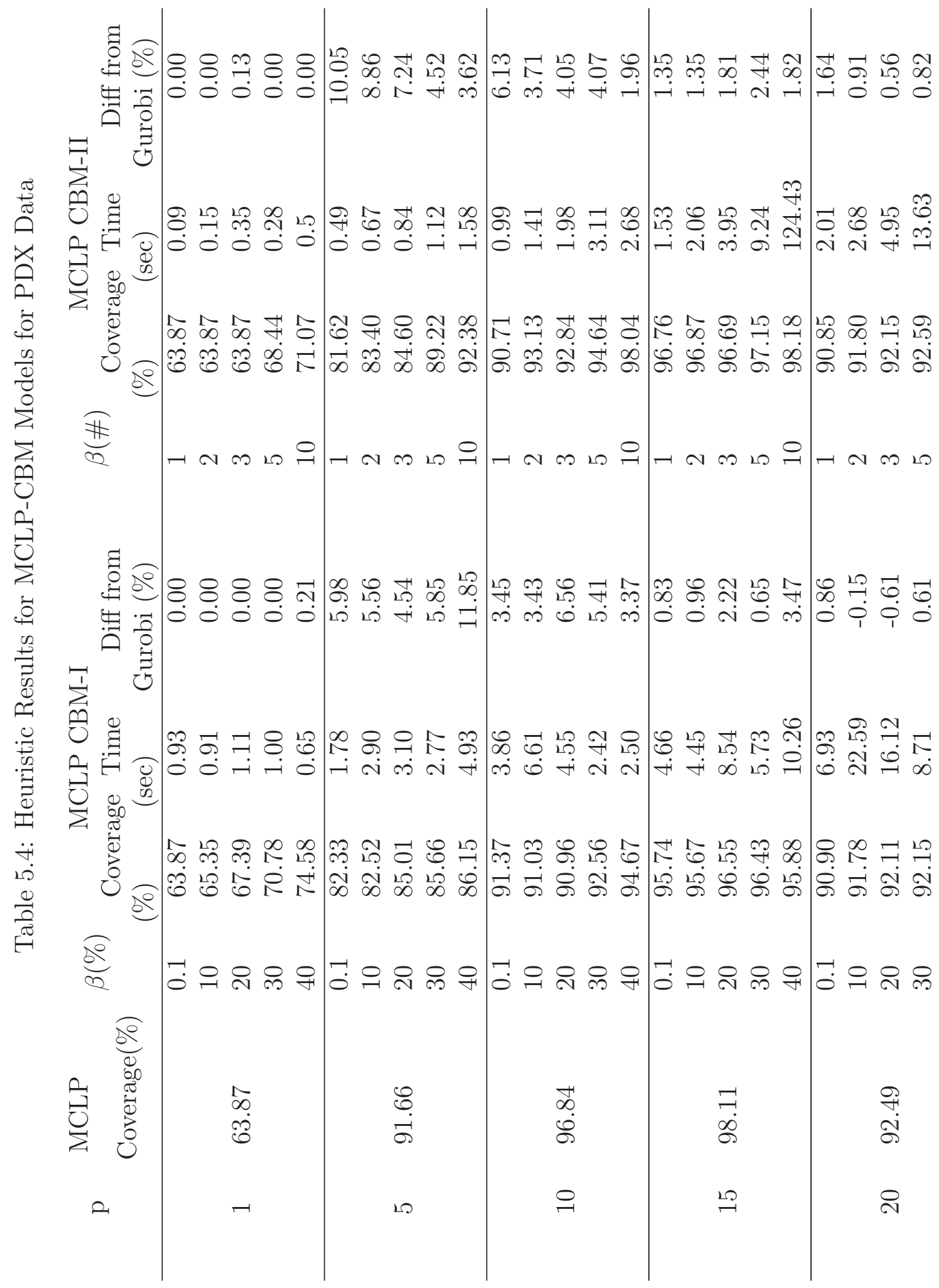




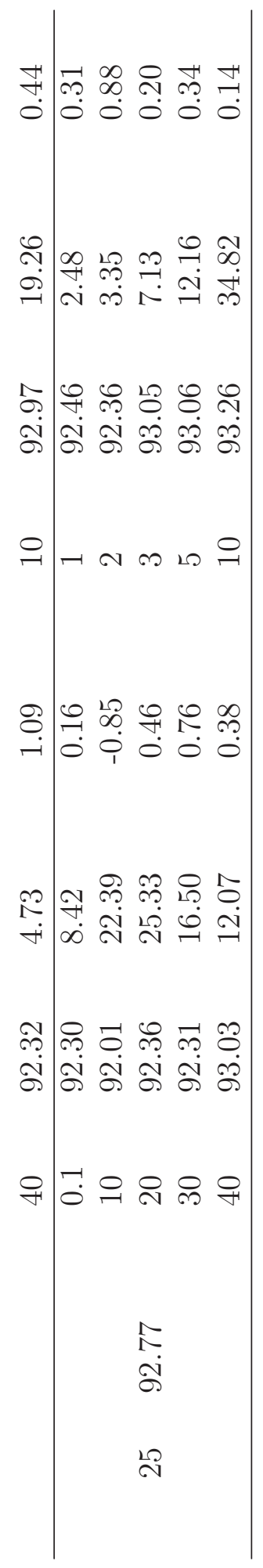


Table 5.5: Heuristic Performance

\begin{tabular}{cccccc} 
Dataset & Average & Median & \multicolumn{2}{c}{ Maximum Minimum } \\
\hline \multirow{2}{*}{ J. E. Beasley } & $\begin{array}{c}\text { Solving Time (sec) } \\
\text { Difference in } \\
\text { Solution (\%) }\end{array}$ & 0.73 & 0.83 & 7.37 & 0.35 \\
\hline \multirow{2}{*}{ PDX } & $\begin{array}{c}\text { Solving Time (sec) } \\
\text { Difference in } \\
\text { Solution (\%) }\end{array}$ & 7.96 & 3.60 & 6.10 & -3.44 \\
\hline
\end{tabular}

\subsection{EMS Applications/MEXSLP Models Analysis}

The same Portland Metropolitan data provided by Chauhan et al. (2019) and explained in section 5.1.1 is used for the study in this section. Demand $w_{i}$ are estimated as explained in section 5.1.1. The probability of survival $s_{i j}$ is calculated by the approach in De Maio et al. (2003) using the equation 4.1.2. The same euclidean distance matrix $d_{i j}$ in the previous section is used as travel time $t_{i j}$ matrix in this study. The travel time threshold $T$ is assumed to be 20 minutes, and $q$ is assumed to be 0.2 , i.e., the EMS units are busy $20 \%$ of the time.

The objective function of MEXSLP models calculates the Aggregated Probability of Survival (APS) of the entire population in the service region. Table 5.6 provides the APS and Gurobi gap values for three models: regular MEXSLP, MEXSLP-CBM-I, and MEXSLP-CBM-II models. In this analysis, all three models are tested with several combinations of $p$ and $\beta$. The number of EMS units to be located $p$ varies from 10 to 40 in multiples of 10 . The $\beta$ parameter used in calculating CBM is considered to be $1 \%, 5 \%, 10 \%$, or $20 \%$. 
Table 5.6: MEXSLP Models - Objective and GAP Values.

\begin{tabular}{|c|c|c|c|c|c|c|}
\hline \multirow{2}{*}{$\mathrm{p}$} & \multirow{2}{*}{$\beta(\%)$} & MEXSLP & \multicolumn{2}{|c|}{ MEXSLP-CBM-I } & \multicolumn{2}{|c|}{ MEXSLP-CBM-II } \\
\hline & & APS & APS & $\begin{array}{c}\text { Gurobi } \\
\text { Gap (\%) }\end{array}$ & APS & $\begin{array}{c}\text { Gurobi } \\
\text { Gap }(\%)\end{array}$ \\
\hline \multirow{3}{*}{10} & 1 & \multirow{3}{*}{46693.07} & 38501.72 & 0 & 38501.72 & 0 \\
\hline & 5 & & 38501.72 & 0 & 38501.72 & 0 \\
\hline & 10 & & 38501.72 & 0 & 38501.72 & 0 \\
\hline \multirow{4}{*}{20} & 1 & \multirow{4}{*}{55945.71} & 53605.63 & 0 & 53605.63 & 0 \\
\hline & 5 & & 53605.63 & 0 & 53605.63 & 0 \\
\hline & 10 & & 51783.79 & 0 & 51783.79 & 0 \\
\hline & 20 & & 40444.99 & 0.23 & 40444.99 & 0.27 \\
\hline \multirow{4}{*}{30} & 1 & \multirow{4}{*}{60578.51} & 59869.20 & 0 & 59869.20 & 0 \\
\hline & 5 & & 59213.22 & 0 & 59213.22 & 0 \\
\hline & 10 & & 57313.36 & 0.02 & 57313.36 & 0.05 \\
\hline & 20 & & 44351.81 & 4.12 & 44364.37 & 3.4 \\
\hline \multirow{4}{*}{40} & 1 & \multirow{4}{*}{63121.26} & 62820.19 & 0 & 62820.19 & 0 \\
\hline & 5 & & 62502.08 & 0 & 62502.08 & 0 \\
\hline & 10 & & 61407.09 & 0.05 & 61407.09 & 0.02 \\
\hline & 20 & & 49084.27 & 7.23 & 50121.77 & 4.11 \\
\hline
\end{tabular}

Observing table 5.6, the APS and $\beta$ appear to be inversely proportional.

Greater the $\beta$, the greater the reduction in APS. To maximize its objective, a MEXSLP model prioritizes assigning more EMS units to urban areas where population density is high. However, the CBM constraint forces some EMS units to be assigned near suburbs to improve equity. So, as the $\beta$ increases, more and more EMS units are assigned to suburbs, resulting in lower APS value. MEXSLP model is not like the MCLP model; this does not consider a demand to be covered in a binary way. So, even with very low $\beta$ any MEXSLP-CBM models need not give the same result as the MEXSLP model.

The Gurobi optimization solver solved test runs of all three MEXSLP models in few minutes, with gap values being less than $10 \%$. It is to be noted that the 
models were tested with even higher values of $\beta$, but they either resulted in higher gap values or turned infeasible. This infeasibility occurs because maximizing coverage always opposes maximizing equity. So, using a large $\beta$ parameter is not advisable.

Figure 5.2-5.7 shows the facility locations where EMS units are placed for MEXSLP, MEXSLP-CBM-I, and MEXSLP-CBM-II models. These solutions are obtained by solving models using Gurobi. In these figures, blue dots represent demand points and red stars represent open facilities, and the size of red star is corresponding to number of EMS units at that site.

Figures 5.2 and 5.5 show the locations of EMS units when a regular MEXSLP model is solved to locate 20 and 40 units, respectively. When $\beta$ is low, both the CBM incorporated MEXSLP models, give the exact same solution. Such solutions of MEXSLP-CBM-I and MEXSLP-CBM-II are plotted and shown in figures 5.3 and 5.6, when $p=20$ and $p=40$, respectively. Similarly, when $p$ is low, both these models provide same solutions even with higher $\beta$ values. Figure 5.4 shows such a result of these models with $p=20$ and $\beta=20 \%$. However, when both $p$ and $\beta$ are high, MEXSLP-CBM-I and MEXSLP-CBM-II models provide different solutions and these results are shown in figure 5.7. 


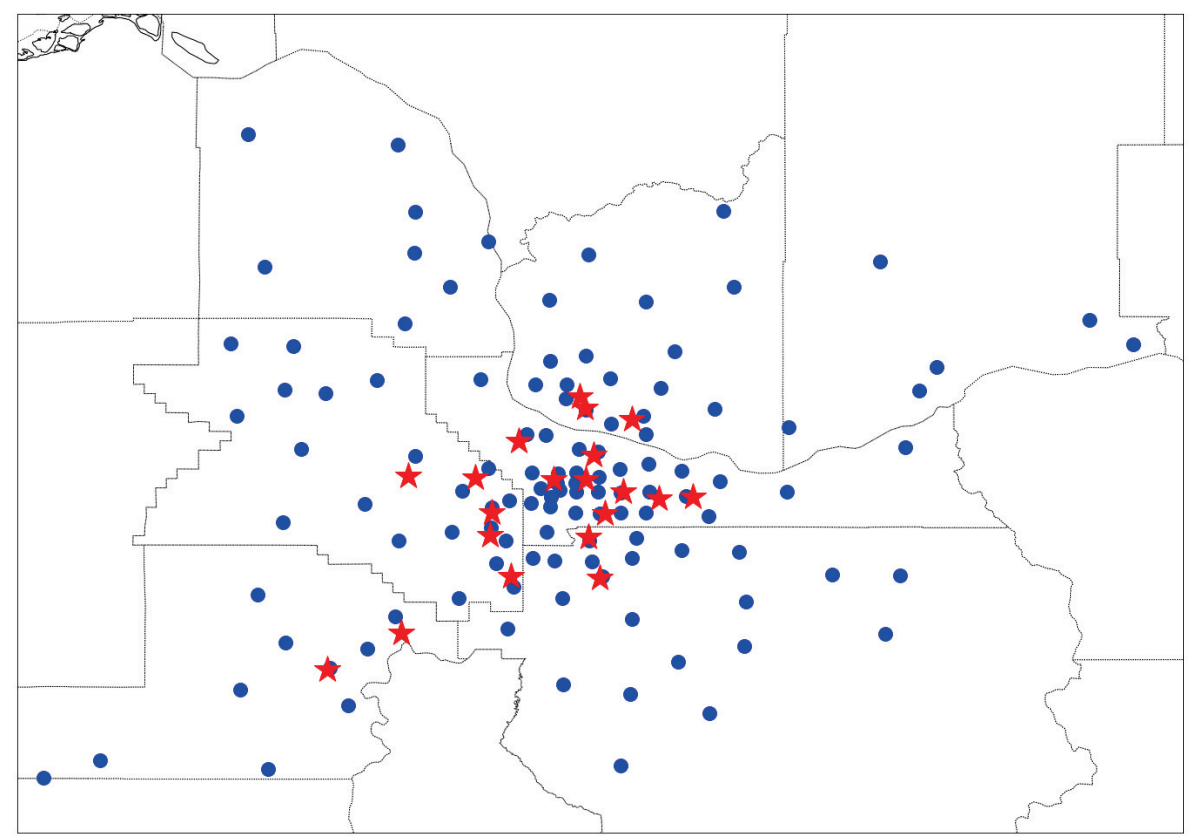

Figure 5.2: MEXSLP solution $(p=20)$

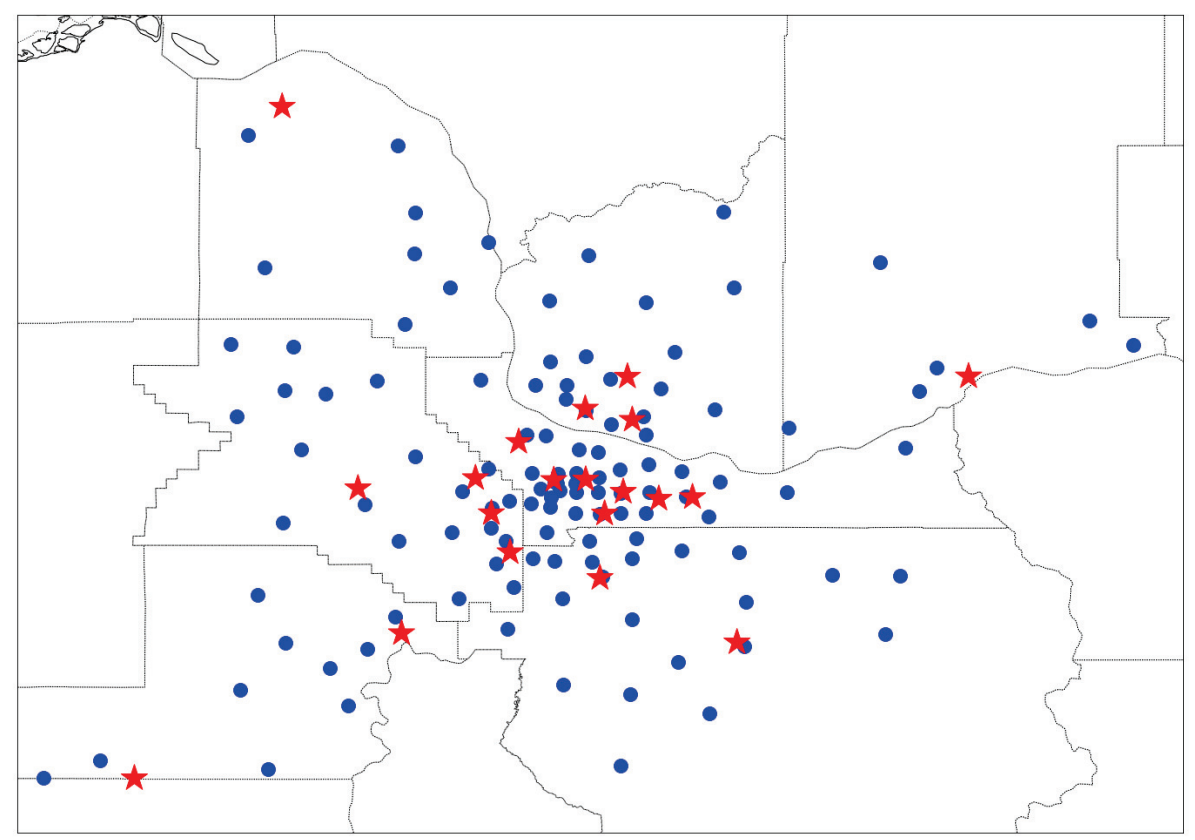

Figure 5.3: MEXSLP CBM-I and MEXSLP CBM-II solutions ( $p=20$ and $\beta=1 \%$ ) 


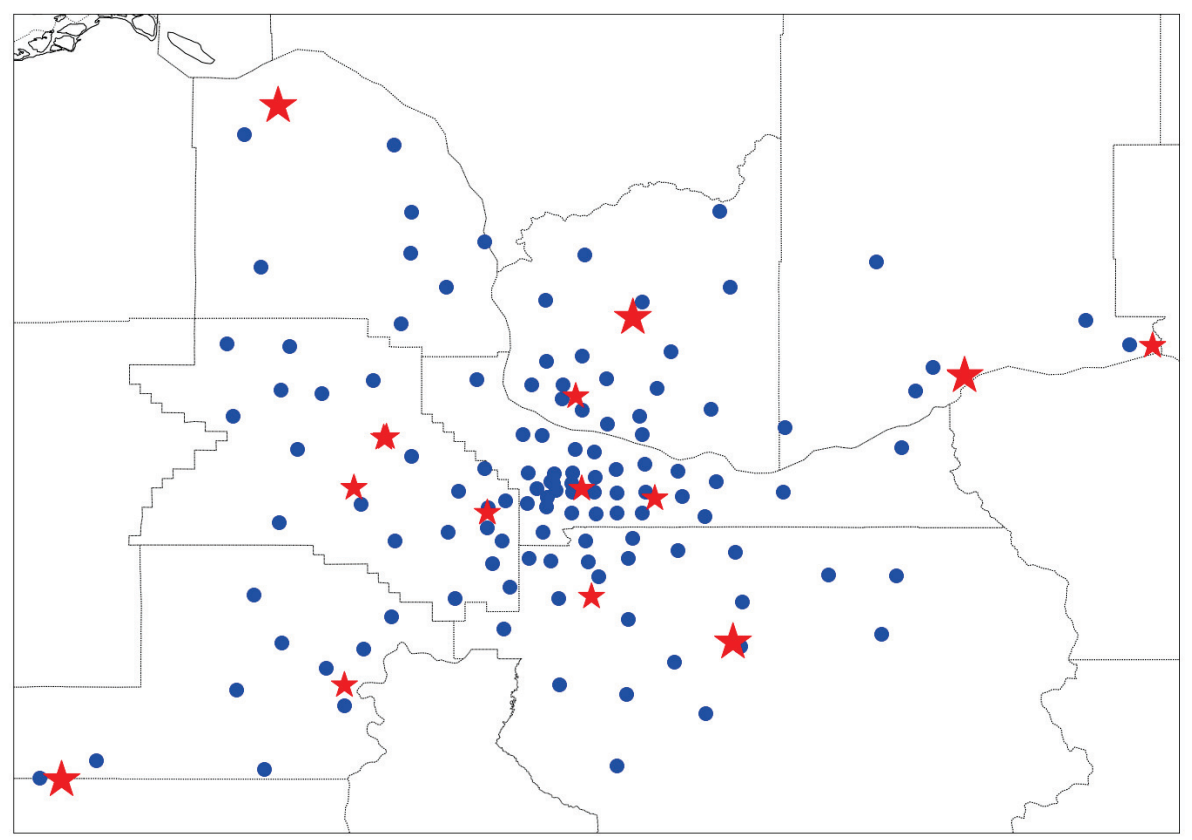

Figure 5.4: MEXSLP CBM-I and MEXSLP CBM-II solutions ( $p=20$ and $\beta=20 \%$ ). Size of star corresponds to EMS units at that location.

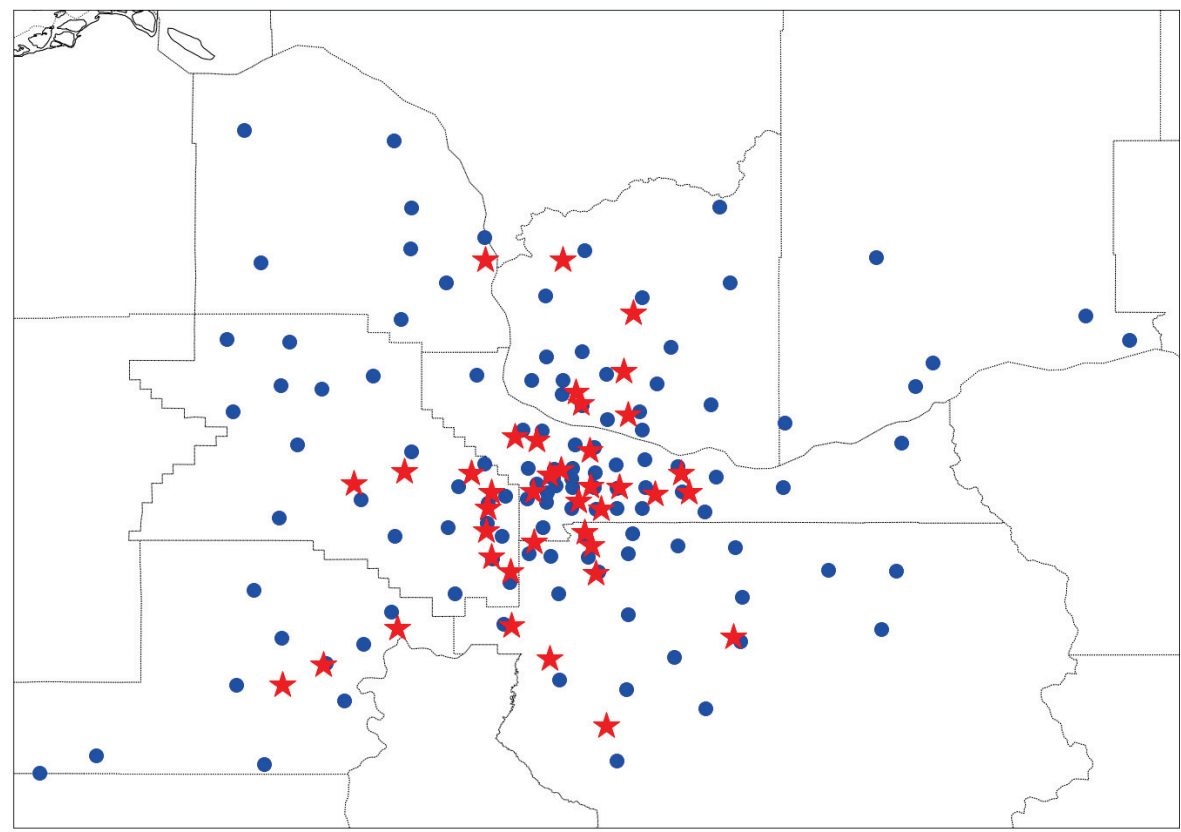

Figure 5.5: MEXSLP solution $(p=40)$. 


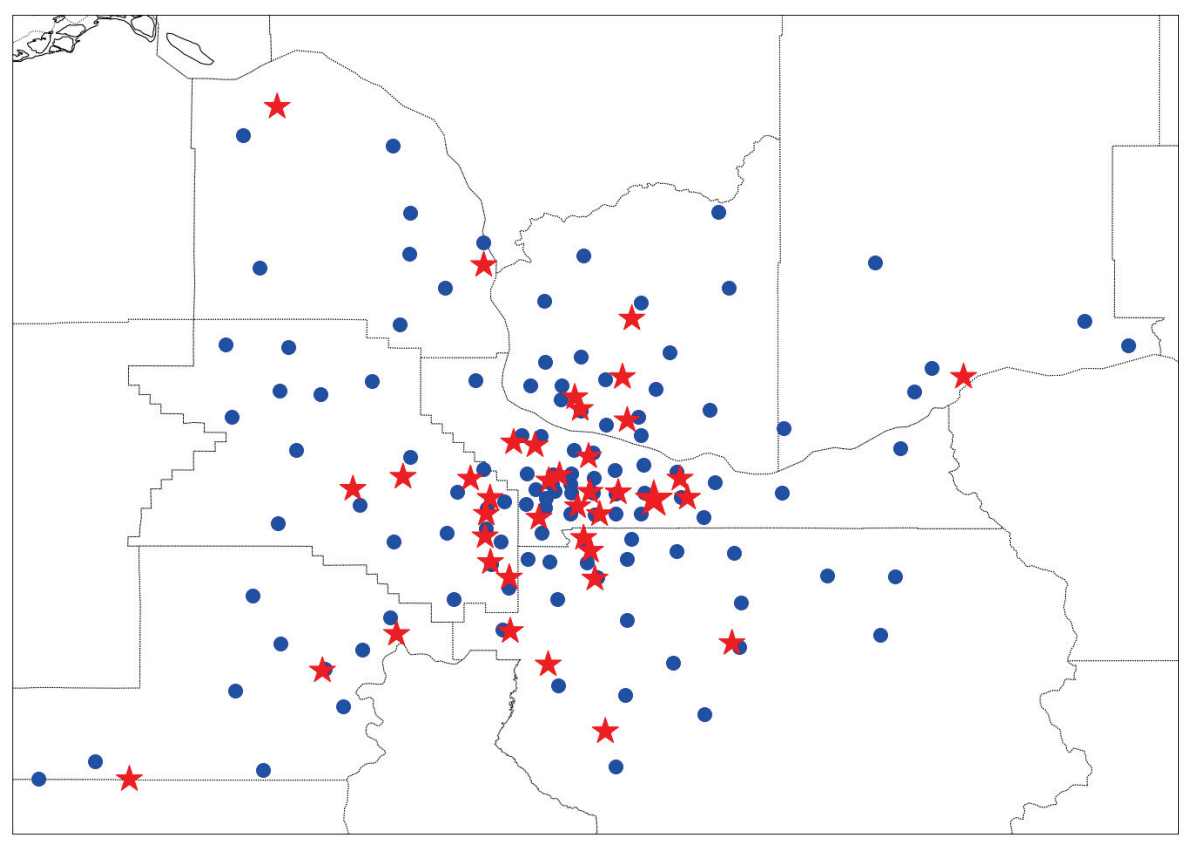

Figure 5.6: MEXSLP CBM-I and MEXSLP CBM-II solutions ( $p=40$ and $\beta=1 \%$ ) 


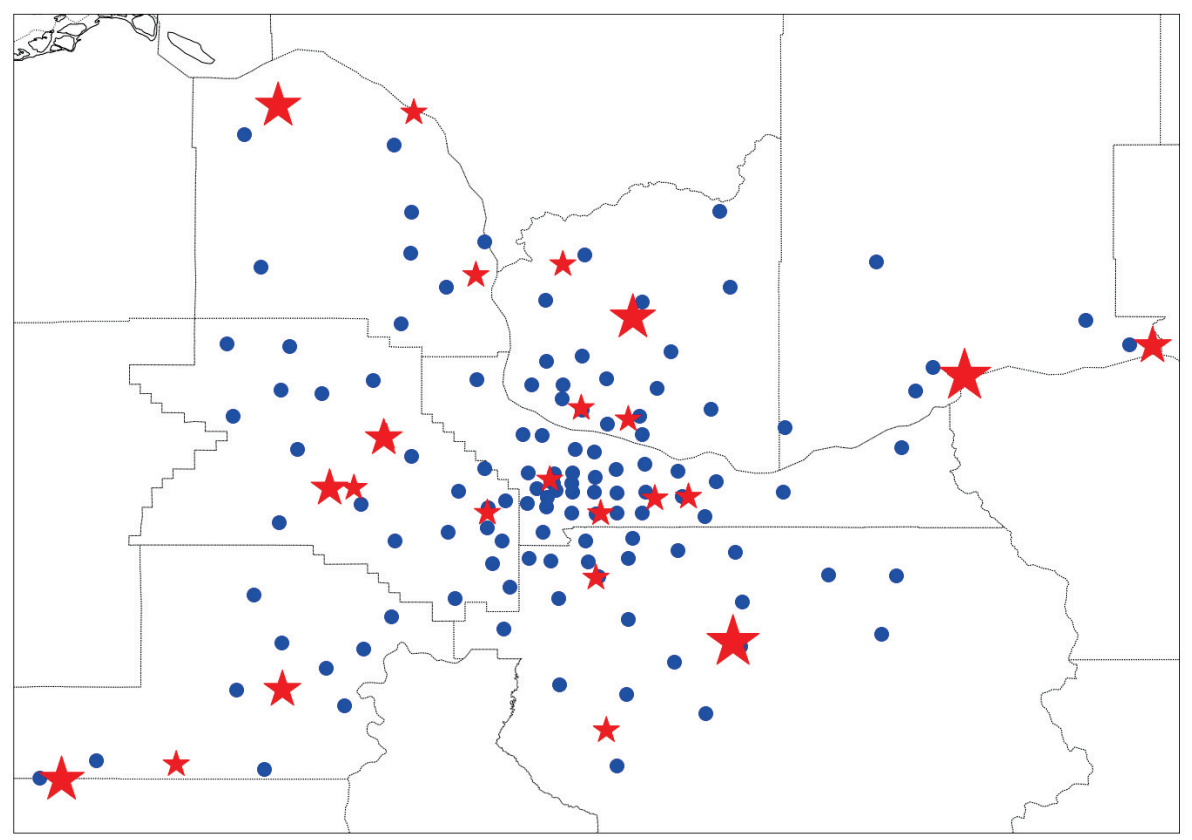

(a) MEXSLP CBM-I

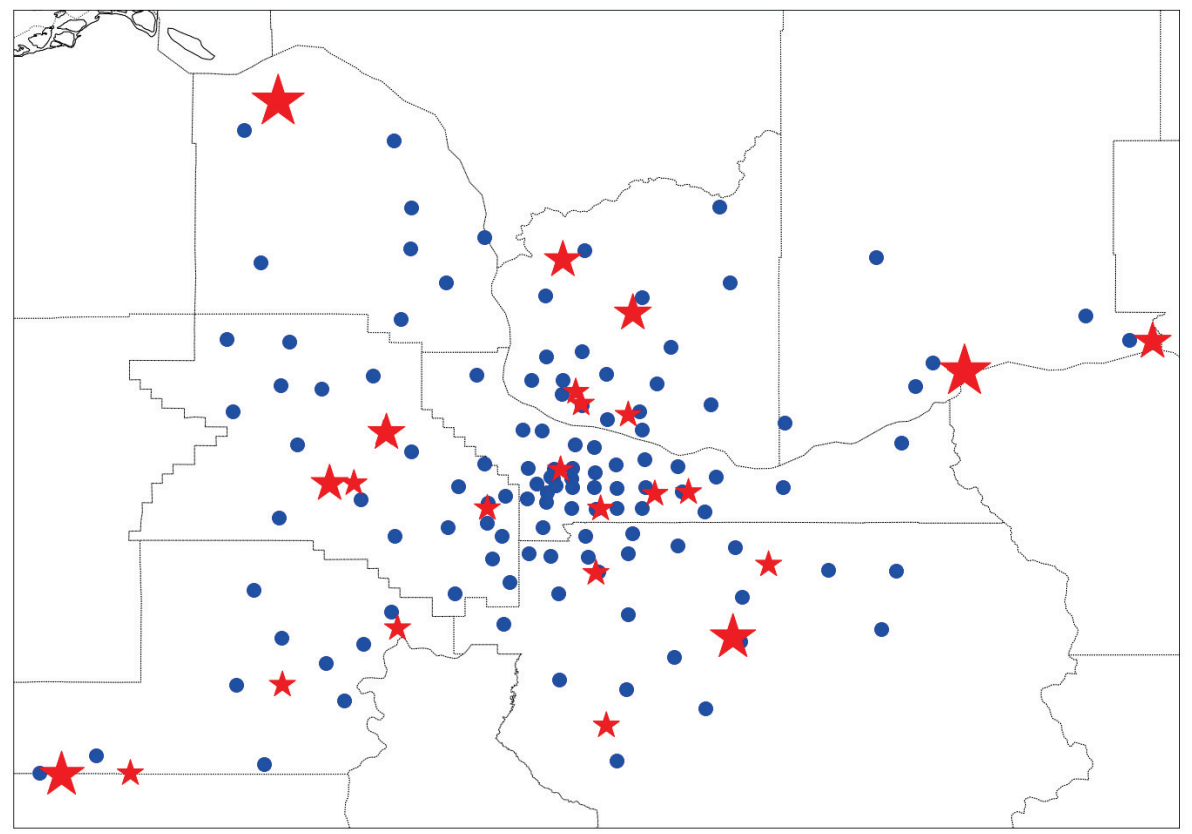

(b) MEXSLP CBM-II

Figure 5.7: MEXSLP models solutions ( $p=40$ and $\beta=20 \%$ ). Size of star corresponds to EMS units at that location.

In MEXSLP model, if there are $n$ EMS units, every unit has a priority num- 
Table 5.7: MEXSLP Model Travel Time Statistics.

\begin{tabular}{cccccccc}
$\mathrm{p}$ & Min & Max & Median & Mean & IQR & SD & $\begin{array}{c}\text { Dispersion } \\
\text { Index }\end{array}$ \\
\hline 10 & 0.21 & 61.46 & 6.04 & 12.07 & 17.19 & 12.16 & 12.26 \\
20 & 0.21 & 56.66 & 4.27 & 9.93 & 12.93 & 11.57 & 13.48 \\
30 & 0.21 & 67.15 & 3.59 & 8.79 & 9.61 & 12.31 & 17.24 \\
40 & 0.21 & 60.98 & 3.34 & 8.36 & 7.32 & 11.87 & 16.83
\end{tabular}

Table 5.8: MEXSLP-CBM-I Model Travel Time Statistics.

\begin{tabular}{|c|c|c|c|c|c|c|c|c|}
\hline $\mathrm{p}$ & $\beta(\%)$ & Min & Max & Median & Mean & IQR & $\mathrm{SD}$ & $\begin{array}{l}\text { Dispersior } \\
\text { Index }\end{array}$ \\
\hline \multirow{3}{*}{10} & 1 & 0.48 & 19.72 & 6.20 & 7.93 & 8.60 & 5.46 & 3.76 \\
\hline & 5 & 0.48 & 19.99 & 6.20 & 8.08 & 9.04 & 5.63 & 3.92 \\
\hline & 10 & 0.48 & 19.72 & 6.20 & 7.93 & 8.60 & 5.46 & 3.76 \\
\hline \multirow{4}{*}{20} & 1 & 0.21 & 19.72 & 4.17 & 6.52 & 8.95 & 5.58 & 4.77 \\
\hline & 5 & 0.21 & 19.72 & 4.17 & 6.52 & 8.95 & 5.58 & 4.77 \\
\hline & 10 & 0.21 & 25.61 & 4.33 & 6.84 & 8.34 & 6.19 & 5.61 \\
\hline & 20 & 0.54 & 32.47 & 5.26 & 7.13 & 6.43 & 5.68 & 4.52 \\
\hline \multirow{4}{*}{30} & 1 & 0.21 & 19.72 & 3.57 & 5.60 & 6.79 & 5.29 & 5.01 \\
\hline & 5 & 0.21 & 28.59 & 3.57 & 5.37 & 5.91 & 5.34 & 5.30 \\
\hline & 10 & 0.21 & 32.14 & 3.77 & 6.24 & 6.24 & 6.58 & 6.95 \\
\hline & 20 & 0.21 & 36.70 & 5.15 & 7.70 & 8.37 & 7.05 & 6.45 \\
\hline \multirow{4}{*}{40} & 1 & 0.21 & 19.72 & 3.48 & 5.30 & 6.57 & 5.27 & 5.24 \\
\hline & 5 & 0.21 & 28.59 & 3.34 & 5.46 & 6.30 & 5.90 & 6.38 \\
\hline & 10 & 0.21 & 36.42 & 3.54 & 5.36 & 5.98 & 5.88 & 6.44 \\
\hline & 20 & 0.21 & 57.82 & 4.33 & 6.66 & 5.53 & 7.57 & 8.59 \\
\hline
\end{tabular}


Table 5.9: MEXSLP-CBM-II Model Travel Time Statistics.

\begin{tabular}{ccccccccc}
$\mathrm{p}$ & $\beta(\%)$ & Min & Max & Median & Mean & IQR & SD & $\begin{array}{c}\text { Dispersion } \\
\text { Index }\end{array}$ \\
\hline \multirow{3}{*}{10} & 1 & 0.48 & 19.99 & 6.20 & 8.08 & 9.04 & 5.63 & 3.92 \\
& 5 & 0.48 & 19.99 & 6.20 & 8.08 & 9.04 & 5.63 & 3.92 \\
& 10 & 0.48 & 19.72 & 6.20 & 7.93 & 8.60 & 5.46 & 3.76 \\
\hline \multirow{4}{*}{20} & 1 & 0.21 & 19.72 & 4.17 & 6.52 & 8.95 & 5.58 & 4.77 \\
& 5 & 0.21 & 19.72 & 4.17 & 6.52 & 8.95 & 5.58 & 4.77 \\
& 10 & 0.21 & 25.61 & 4.33 & 6.84 & 8.34 & 6.19 & 5.61 \\
& 20 & 0.54 & 31.45 & 5.22 & 7.02 & 6.48 & 5.37 & 4.11 \\
\hline \multirow{3}{*}{30} & 1 & 0.21 & 19.72 & 3.57 & 5.60 & 6.79 & 5.29 & 5.01 \\
& 5 & 0.21 & 28.59 & 3.57 & 5.37 & 5.91 & 5.34 & 5.30 \\
& 10 & 0.21 & 25.96 & 3.71 & 5.80 & 6.11 & 5.82 & 5.84 \\
& 20 & 0.21 & 40.68 & 4.33 & 6.68 & 5.32 & 6.09 & 5.56 \\
\hline \multirow{4}{*}{40} & 1 & 0.21 & 19.72 & 3.48 & 5.30 & 6.57 & 5.27 & 5.24 \\
& 5 & 0.21 & 28.59 & 3.34 & 5.46 & 6.30 & 5.90 & 6.38 \\
& 10 & 0.21 & 46.84 & 3.54 & 5.52 & 5.55 & 6.75 & 8.26 \\
& 20 & 0.21 & 29.83 & 4.23 & 5.92 & 5.24 & 5.24 & 4.63
\end{tabular}

ber assigned to it with respect to a demand point. Now we will analyze the travel times of all the first priority EMS routes. Table 5.7 provide multiple statistical measures for travel times of the first priority routes for a regular MEXSLP model. Tables 5.8 and 5.9 provide the same for MEXSLP-CBM-I and MEXSLP-CBM-II models.

Comparing tables 5.7-5.9, the reduction in maximum travel time and Interquartile range due to CBM is higher when $p$ is low. And, as $p$ increases the effect of CBM on model decreases. Incorporation of CBM in MEXSLP model has no significant effect on median or mean travel time. In terms of dispersion and SD, both the MEXSLP-CBM models appear to be more effective for lower values of $\beta$ and $p$. Overall, incorporating CBM into MEXSLP did help in reducing skewness 
and shortening the tails of travel times. Though both the MEXSLP-CBM models did perform good in reducing travel times and dispersion, while MEXSLP-CBM-II model seems to perform even better and consistent.

Figure 5.8 shows the distribution of travel times of the first priority routes obtained from the solutions of MEXSLP, MEXSLP-CBM-I, and MEXSLP-CBMII models. Here it is clear that density of travel times above 20 minutes has reduced in CBM incorporated models. Correspondingly, the density of travel times less than 20 minutes has increased.

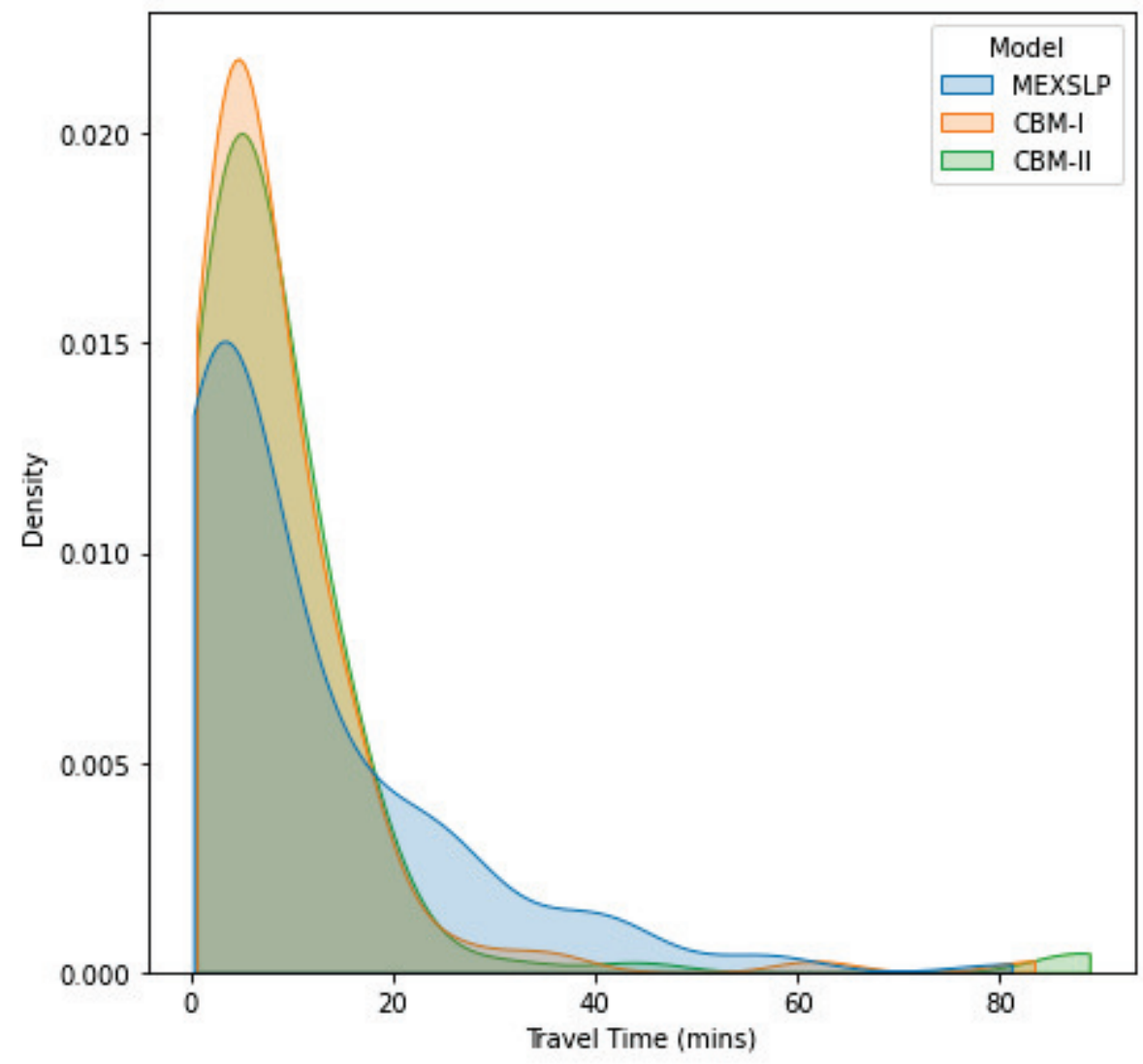

Figure 5.8: Distribution of Travel Times from facilities to demand ( $p=20$ and $\beta=20 \%)$ 


\subsection{Summary}

In this chapter, computational analyses are performed on four CBM models developed in chapters 3 and 4 . First, the two MCLP-CBM models' computational performance from chapter 3 is compared to a regular MCLP by solving them using a state-of-the-art MIP solver. MCLP-CBM-I model is defined such that the CBM part in it is calculated using $\beta \%$ of demands served by such facility. MCLP-CBM-II model is defined such that the CBM is calculated using $\beta$ number of demands served by a facility. Both the MCLP-CBM models are tested with two different types of datasets, one taken from Chauhan et al. (2019) and the other from Osman and Christofides (1994). Results analyses of both the datasets show that incorporating CBM has helped MCLP utilize the excess capacity by assigning it to appropriate demand points outside coverage radius. MCLP-CBM-II model always showed better convergence over the MCLP-CBM-I model.

Though MCLP-CBM models provided better coverage, their solutions are less reliable due to their higher gap values from MIP solver. To overcome the issue of convergence and solve the models faster, an Enhanced Greedy (EG) based heuristic was proposed. Compared to the MIP solver, EG heuristic achieves better quality solutions in a very short time. This has been observed and verified with both datasets.

Next, the computational performance of the two MEXSLP-CBM models 
from chapter 4 are compared to a regular MEXSLP model. MEXSLP is a model that specially focuses on locating emergency supplies or EMS units. Due to the population density of urban areas, MEXSLP model locates the majority of the EMS units closer to urban areas, affecting the quality of service in suburban regions. The incorporation of CBM into the MEXSLP has resulted in improving the quality of service provided for suburban regions. Unlike the other nonlinear EMS models incorporating equity, MEXSLP-CBM models in this study are linear and easy to solve using a MIP solver. 


\section{Conclusions}

For any facility location problem, the primary goal is to locate facilities while optimizing specific objectives and satisfying constraints. Facility location problems such as Maximum Covering Problem (MCLP) have been popular to locate facilities such as postal services, police, emergency services, garbage disposal, and more. A classical MCLP is designed such that a demand point is considered covered if it has an open facility within the coverage radius and unserved if it is outside the radius. There is no hardbound coverage radius for public services such as postal or medical. In such cases, coverage radius is only used to maintain a minimum level of service while serving the maximum demand possible.

Similarly, in FLP focusing on EMS, the assumption of a patient being covered only when it is in the coverage radius is not practical. When it comes to medical services, successful coverage is always a just probability. If a patient stays closer to an EMS unit, their probability of survival is higher, and when they stay far, their probability of survival is lower. So, irrespective of their location, in EMS, all demands are served based on availability. MEXSLP is an excellent example of such a model whose objective is to maximize the aggregated sum of the survival probability of all demand points.

With a single objective of maximizing coverage, models tend to locate fa- 
cilities closer to urban areas having a dense population, leaving minimal to no resources available for suburban and rural regions. Even if more EMS units are made available, these models tend to concentrate units in favor of the denser population unless $100 \%$ coverage is possible. Maximizing coverage could be an efficient way of utilizing resources, but it is not equitable.

In literature, there have been various studies with various approaches and measures to obtain equity. However, there are not models which are linear and incorporate equity. So, in our study, we chose CBM as the measure for equity in the MEXSLP model. Also, we use the same CBM to provide soft or flexible bounds on the coverage radius of MCLP models. As explained in earlier chapters, CBM has few unique characteristics, allowing us to implement it in a MILP model with as limited constraints and variables as possible.

In this study, four models were proposed and developed, incorporated with CBM: MCLP-CBM-I, MCLP-CBM-II, MEXSLP-CBM-I, and MEXSLP-CBM-II. Though both the MCLP-CBM models have the same objectives, the way CBM is defined and calculated is different. MCLP-CBM-I model uses the standard definition of CBM where $\beta$ is a percentage. In the MCLP-CBM-II model, the idea of CBM is similar, but instead of percentage, $\beta$ is defined as an integer. Defining $\beta$ in this way made the model computationally efficient. Besides, with $\beta$ being integer, the calculation of CBM is relatively simple and straight.

Overall, both the MCLP-CBM models increase coverage by assigning de- 
mands outside the coverage radius to facilities having excess capacity or resources. An enhanced greedy-based heuristic is proposed for solving the MCLP-CBM models to reduce run-time and improve convergence within solutions. The developed enhanced greedy heuristic provides coverage almost as good as a standard MIP solver while saving more than $95 \%$ of time.

For EMS applications, two linear MEXSLP models were proposed while incorporating equity using CBM. Being linear, both these can be easily solved by any standard MIP solver. Both the MEXSLP-CBM models have a different approach for estimating CBM. Both models are efficient in incorporating equity and reducing the response time for suburban regions, giving them a higher probability of survival in case of emergencies. Geo-plots in section 5.3 show the capability of these models to locate EMS units across the service area sparsely are, providing equity concerning the level of service in suburban regions.

The models developed in this study assume only one type of EMS unit, a single type of patient severity, and do not account for vehicle routing. All of these are the limitations of this study and can be potential directions for future research. 


\section{Bibliography}

M. Albareda-Sambola, E. Fernández, and G. Laporte. The capacity and distance constrained plant location problem. Computers \& Operations Research, 36(2): 597-611, 2009.

American Community Survey. U.S. Census Bureau, 2014-2018

American Community Survey 5-Year Estimates, Table S1701, 2020.

https://data.census.gov/cedsci/, last accessed on 6/15/2020.

C. Araz, H. Selim, and I. Ozkarahan. A fuzzy multi-objective covering-based vehicle location model for emergency services. Computers $\&$ Operations Research, 34(3):705-726, 2007.

R. Aringhieri, M. E. Bruni, S. Khodaparasti, and J. T. van Essen. Emergency medical services and beyond: Addressing new challenges through a wide literature review. Computers \& Operations Research, 78:349-368, 2017.

M. O. Ball and F. L. Lin. A reliability model applied to emergency service vehicle location. Operations research, 41(1):18-36, 1993.

D. Bandara, M. E. Mayorga, and L. A. McLay. Optimal dispatching strategies for emergency vehicles to increase patient survivability. International Journal of Operational Research, 15(2):195-214, 2012.

M. Barbati and C. Piccolo. Equality measures properties for location problems. Optimization Letters, 10(5):903-920, 2016.

O. Baron, O. Berman, D. Krass, and Q. Wang. The equitable location problem on the plane. European Journal of Operational Research, 183(2):578-590, 2007.

R. Batta, J. M. Dolan, and N. N. Krishnamurthy. The maximal expected covering location problem: Revisited. Transportation science, 23(4):277-287, 1989.

V. Bélanger, A. Ruiz, and P. Soriano. Recent optimization models and trends in location, relocation, and dispatching of emergency medical vehicles. European Journal of Operational Research, 272(1):1-23, 2019. 
G. N. Berlin. Facility location and vehicle allocation for provision of an emergency service. 1974.

O. Berman and R. Huang. The minimum weighted covering location problem with distance constraints. Computers \& Operations Research, 35(2):356-372, 2008.

O. Berman and D. Krass. 11 facility location problems with stochastic demands and congestion. Facility location: Applications and theory, 329, 2001.

O. Berman and D. Krass. The generalized maximal covering location problem. Computers \& Operations Research, 29(6):563-581, 2002.

O. Berman, Z. Drezner, and D. Krass. Generalized coverage: New developments in covering location models. Computers $\& 3$ Operations Research, 37(10):1675-1687, 2010 .

R. Boujemaa, A. Jebali, S. Hammami, A. Ruiz, and H. Bouchriha. A stochastic approach for designing two-tiered emergency medical service systems. Flexible Services and Manufacturing Journal, 30(1):123-152, 2018.

C. C. Branas and C. S. Revelle. An iterative switching heuristic to locate hospitals and helicopters. Socio-Economic Planning Sciences, 35(1):11-30, 2001.

L. Brotcorne, G. Laporte, and F. Semet. Ambulance location and relocation models. European journal of operational research, 147(3):451-463, 2003.

S. Chanta. Improving quality of service in ems systems by reducing disparities between service zones. 2011.

S. Chanta, M. E. Mayorga, M. E. Kurz, and L. A. McLay. The minimum penvy location problem: a new model for equitable distribution of emergency resources. IIE Transactions on Healthcare Systems Engineering, 1(2):101-115, 2011

S. Chanta, M. E. Mayorga, and L. A. McLay. Improving emergency service in rural areas: a bi-objective covering location model for ems systems. Annals of Operations Research, 221(1):133-159, 2014a. 
S. Chanta, M. E. Mayorga, and L. A. McLay. The minimum p-envy location problem with requirement on minimum survival rate. Computers $\&$ Industrial Engineering, 74:228-239, 2014b.

A. G. Chapman and J. E. Mitchell. A fair division approach to humanitarian logistics inspired by conditional value-at-risk. Annals of Operations Research, 262(1):133-151, 2018.

D. Chauhan, A. Unnikrishnan, and M. Figliozzi. Maximum coverage capacitated facility location problem with range constrained drones. Transportation Research Part C: Emerging Technologies, 99:1-18, 2019.

G. Chen, M. S. Daskin, Z.-J. M. Shen, and S. Uryasev. The $\alpha$-reliable meanexcess regret model for stochastic facility location modeling. Naval Research Logistics (NRL), 53(7):617-626, 2006.

S.-H. Cho, H. Jang, T. Lee, and J. Turner. Simultaneous location of trauma centers and helicopters for emergency medical service planning. Operations Research, 62(4):751-771, 2014.

C. Chung, D. Schilling, and R. Carbone. The capacitated maximal covering problem: A heuristic. In Proceedings of Fourteenth Annual Pittsburgh Conference on Modeling and Simulation, volume 1983, pages 1423-1428, 1983.

R. Church and C. ReVelle. The maximal covering location problem. In Papers of the Regional Science Association, volume 32, pages 101-118. Springer-Verlag, 1974.

P. Coelho. Linearization of the product of two variables. Canada Research Chair in Integrated Logistics, 2013.

I. Correia and F. Saldanha-da Gama. Facility location under uncertainty. In Location science, pages 185-213. Springer, 2019.

J. Current, H. Min, and D. Schilling. Multiobjective analysis of facility location decisions. European journal of operational research, 49(3):295-307, 1990.

J. R. Current and J. E. Storbeck. Capacitated covering models. Environment and planning B: planning and Design, 15(2):153-163, 1988. 
M. S. Daskin. A maximum expected covering location model: formulation, properties and heuristic solution. Transportation science, 17(1):48-70, 1983.

M. S. Daskin. Network and discrete location: models, algorithms, and applications. John Wiley \& Sons, 2011.

M. S. Daskin and E. H. Stern. A hierarchical objective set covering model for emergency medical service vehicle deployment. Transportation Science, 15(2): $137-152,1981$.

M. S. Daskin, K. Hogan, and C. ReVelle. Integration of multiple, excess, backup, and expected covering models. Environment and Planning B: Planning and Design, 15(1):15-35, 1988.

M. S. Daskin, S. M. Hesse, and C. S. Revelle. $\alpha$-reliable p-minimax regret: A new model for strategic facility location modeling. Location science, 5(4):227-246, 1997.

V. J. De Maio, I. G. Stiell, G. A. Wells, D. W. Spaite, O. P. A. L. S. S. Group, et al. Optimal defibrillation response intervals for maximum out-of-hospital cardiac arrest survival rates. Annals of emergency medicine, 42(2):242-250, 2003.

T. Drezner, Z. Drezner, and J. Guyse. Equitable service by a facility: Minimizing the gini coefficient. Computers $\&$ operations research, 36(12):3240-3246, 2009.

T. Drezner, Z. Drezner, and B. Hulliger. The quintile share ratio in location analysis. European Journal of Operational Research, 238(1):166-174, 2014.

E. Erkut and S. Neuman. A multiobjective model for locating undesirable facilities. Annals of operations Research, 40(1):209-227, 1992.

E. Erkut, A. Ingolfsson, and G. Erdoğan. Ambulance location for maximum survival. Naval Research Logistics (NRL), 55(1):42-58, 2008.

I. Espejo, A. Marín, J. Puerto, and A. M. Rodríguez-Chía. A comparison of formulations and solution methods for the minimum-envy location problem. Computers \&f Operations Research, 36(6):1966-1981, 2009. 
R. Z. Farahani, N. Asgari, N. Heidari, M. Hosseininia, and M. Goh. Covering problems in facility location: A review. Computers \& Industrial Engineering, 62 (1):368-407, 2012.

C. Filippi, G. Guastaroba, and M. Speranza. Applications of conditional value-atrisk beyond finance: A literature review. In Technical Report 2. University of Brescia, Department of Economics and Management, 2017.

C. Filippi, G. Guastaroba, and M. Speranza. On single-source capacitated facility location with cost and fairness objectives. European Journal of Operational Research, 2019a.

C. Filippi, W. Ogryczak, and M. G. Speranza. Bridging k-sum and cvar optimization in milp. Computers $\& 3$ Operations Research, 105:156-166, 2019b.

C. Filippi, G. Guastaroba, and M. G. Speranza. Conditional value-at-risk beyond finance: a survey. International Transactions in Operational Research, 27(3): 1277-1319, 2020.

M. Gendreau, G. Laporte, and F. Semet. Solving an ambulance location model by tabu search. Location science, 5(2):75-88, 1997.

M. Gendreau, G. Laporte, and F. Semet. A dynamic model and parallel tabu search heuristic for real-time ambulance relocation. Parallel computing, 27(12): 1641-1653, 2001.

M. Gendreau, G. Laporte, and F. Semet. The maximal expected coverage relocation problem for emergency vehicles. Journal of the Operational Research Society, 57(1):22-28, 2006.

A. Ghosh and S. McLaerty. Locating stores in uncertain environments: A scenario planning approach. 1982.

B. C. Grannan, N. D. Bastian, and L. A. McLay. A maximum expected covering problem for locating and dispatching two classes of military medical evacuation air assets. Optimization Letters, 9(8):1511-1531, 2015.

L. Gurobi Optimization. Gurobi optimizer reference manual, 2020. URL http: //www.gurobi.com. 
A. Haghani. Capacitated maximum covering location models: Formulations and solution procedures. Journal of advanced transportation, 30(3):101-136, 1996.

S. Hammami and A. Jebali. Designing modular capacitated emergency medical service using information on ambulance trip. Operational Research, pages 1-20, 2019.

K. Hogan and C. ReVelle. Concepts and applications of backup coverage. Management science, 32(11):1434-1444, 1986.

C. Jagtenberg and A. Mason. Fairness in the ambulance location problem: Maximizing the bernoulli-nash social welfare. Available at SSRN 3536r0\%, 2020.

V. Jayaraman and R. Srivastava. A service logistics model for simultaneous siting of facilities and multiple levels of equipment. Computers $\&$ Operations Research, 22(2):191-204, 1995.

O. Karasakal and E. K. Karasakal. A maximal covering location model in the presence of partial coverage. Computers $\&$ Operations Research, 31(9):1515$1526,2004$.

M. M. Kostreva, W. Ogryczak, and A. Wierzbicki. Equitable aggregations and multiple criteria analysis. European Journal of Operational Research, 158(2): 362-377, 2004.

T. Lee and H. Jang. An iterative method for simultaneously locating trauma centers and helicopters through the planning horizon. Operations research for health care, 19:185-196, 2018.

T. Lee, S.-H. Cho, H. Jang, and J. G. Turner. A simulation-based iterative method for a trauma center - air ambulance location problem. In Proceedings of the 2012 Winter Simulation Conference (WSC), pages 1-12. IEEE, 2012.

M. A. Lejeune and S. Y. Prasad. Effectiveness-equity models for facility location problems on tree networks. networks, 62(4):243-254, 2013.

X. Li, Z. Zhao, X. Zhu, and T. Wyatt. Covering models and optimization techniques for emergency response facility location and planning: a review. Mathematical Methods of Operations Research, 74(3):281-310, 2011. 
Y. Liu, H. Chen, and B. Wu. Controllability of boolean control networks with impulsive effects and forbidden states. Mathematical methods in the Applied Sciences, 37(1):1-9, 2014.

Y. Liu, Z. Li, J. Liu, and H. Patel. A double standard model for allocating limited emergency medical service vehicle resources ensuring service reliability. Transportation research part C: emerging technologies, 69:120-133, 2016.

M. C. López-de-los Mozos and J. A. Mesa. The maximum absolute deviation measure in location problems on networks. European Journal of Operational Research, 135(1):184-194, 2001.

M. B. Mandell. Covering models for two-tiered emergency medical services systems. Location Science, 6(1-4):355-368, 1998.

V. Marianov and C. ReVelle. A probabilistic fire-protection siting model with joint vehicle reliability requirements. Papers in Regional Science, 71(3):217-241, 1992.

V. Marianov and D. Serra. Hierarchical location-allocation models for congested systems. European Journal of Operational Research, 135(1):195-208, 2001.

A. Marín. The discrete facility location problem with balanced allocation of customers. European Journal of Operational Research, 210(1):27-38, 2011.

M. T. Marsh and D. A. Schilling. Equity measurement in facility location analysis: A review and framework. European journal of operational research, 74(1): $1-17,1994$.

A. J. Mason. Simulation and real-time optimised relocation for improving ambulance operations. In Handbook of Healthcare Operations Management, pages 289-317. Springer, 2013.

L. A. McLay. A maximum expected covering location model with two types of servers. IIE Transactions, 41(8):730-741, 2009.

L. A. McLay and M. E. Mayorga. A dispatching model for server-to-customer systems that balances efficiency and equity. Manufacturing $\& 5$ Service Operations Management, 15(2):205-220, 2013. 
S. Narasimhan, H. Pirkul, and D. A. Schilling. Capacitated emergency facility siting with multiple levels of backup. Annals of Operations Research, 40(1): 323-337, 1992.

W. Ogryczak. Inequality measures and equitable approaches to location problems. European Journal of Operational Research, 122(2):374-391, 2000.

W. Ogryczak and M. Zawadzki. Conditional median: a parametric solution concept for location problems. Annals of Operations Research, 110(1-4):167-181, 2002.

Y. Ohsawa, F. Plastria, and K. Tamura. Euclidean push-pull partial covering problems. Computers $\&$ operations research, 33(12):3566-3582, 2006.

R. L. Oliver and W. S. DeSarbo. Response determinants in satisfaction judgments. Journal of consumer research, 14(4):495-507, 1988.

I. H. Osman and N. Christofides. Capacitated clustering problems by hybrid simulated annealing and tabu search. International Transactions in Operational Research, 1(3):317-336, 1994.

H. Pirkul and D. Schilling. The capacitated maximal covering location problem with backup service. Annals of Operations Research, 18(1):141-154, 1989.

H. Pirkul and D. A. Schilling. The siting of emergency service facilities with workload capacities and backup service. Management Science, 34(7):896-908, 1988.

H. Pirkul and D. A. Schilling. The maximal covering location problem with capacities on total workload. Management Science, 37(2):233-248, 1991.

O. A. Prokopyev, N. Kong, and D. L. Martinez-Torres. The equitable dispersion problem. European Journal of Operational Research, 197(1):59-67, 2009.

C. ReVelle and K. Hogan. The maximum availability location problem. Transportation science, 23(3):192-200, 1989.

C. ReVelle and V. Marianov. A probabilistic fleet model with individual vehicle reliability requirements. European Journal of Operational Research, 53(1): 93-105, 1991. 
C. Revelle, J. Schweitzer, and S. Snyder. The maximal conditional covering problem. INFOR: Information Systems and Operational Research, 34(2):77-91, 1996.

C. S. ReVelle and H. A. Eiselt. Location analysis: A synthesis and survey. European journal of operational research, 165(1):1-19, 2005.

C. S. Revelle, H. A. Eiselt, and M. S. Daskin. A bibliography for some fundamental problem categories in discrete location science. European Journal of Operational Research, 184(3):817-848, 2008.

D. Rey, K. Almi'ani, and D. J. Nair. Exact and heuristic algorithms for finding envy-free allocations in food rescue pickup and delivery logistics. Transportation Research Part E: Logistics and Transportation Review, 112:19-46, 2018.

R. T. Rockafellar, S. Uryasev, et al. Optimization of conditional value-at-risk. Journal of risk, 2:21-42, 2000.

T. W. Ruefli and J. E. Storbeck. Behaviorally linked location hierarchies. Environment and Planning B: Planning and Design, 9(3):257-268, 1982.

E. S. Savas. On equity in providing public services. Management Science, 24(8): 800-808, 1978.

D. Schilling, D. J. Elzinga, J. Cohon, R. Church, and C. ReVelle. The team/fleet models for simultaneous facility and equipment siting. Transportation science, 13(2):163-175, 1979.

D. A. Schilling. Strategic facility planning: The analysis of options. Decision Sciences, 13(1):1-14, 1982.

D. A. Schilling. A review of covering problems in facility location. Location Science, 1:25-55, 1993.

E. S. Sheppard. A conceptual framework for dynamic location - allocation analysis. Environment and Planning a, 6(5):547-564, 1974.

L. V. Snyder. Facility location under uncertainty: a review. IIE transactions, 38 (7):547-564, 2006. 
L. V. Snyder, M. S. Daskin, and C.-P. Teo. The stochastic location model with risk pooling. European Journal of Operational Research, 179(3):1221-1238, 2007.

L. V. Snyder, Z. Atan, P. Peng, Y. Rong, A. J. Schmitt, and B. Sinsoysal. Or/ms models for supply chain disruptions: A review. IIE Transactions, 48(2):89-109, 2016.

K. Sudtachat, M. E. Mayorga, and L. A. McLay. Recommendations for dispatching emergency vehicles under multitiered response via simulation. International Transactions in Operational Research, 21(4):581-617, 2014.

C. Toregas and C. ReVelle. Optimal location under time or distance constraints. In Papers of the Regional Science Association, volume 28, pages 131-143. Springer, 1972.

C. Toregas, R. Swain, C. ReVelle, and L. Bergman. The location of emergency service facilities. Operations research, 19(6):1363-1373, 1971.

T. D. Valenzuela, D. J. Roe, S. Cretin, D. W. Spaite, and M. P. Larsen. Estimating effectiveness of cardiac arrest interventions: a logistic regression survival model. Circulation, 96(10):3308-3313, 1997.

T. Van Barneveld, R. D. van der Mei, and S. Bhulai. Compliance tables for an ems system with two types of medical response units. Computers 83 Operations Research, 80:68-81, 2017.

S. Yoon and L. Albert. Dynamic resource assignment for emergency response with multiple types of vehicles. Under review at Operations Research, 2018.

S. Yoon and L. A. Albert. A dynamic ambulance routing model with multiple response. Transportation Research Part E: Logistics and Transportation Review, 133:101807, 2020.

S. Yoon, L. A. Albert, and V. M. White. A stochastic programming approach for locating and dispatching two types of ambulances. Transportation Science, 55 (2):275-296, 2021. 
S.-l. Zhan and N. Liu. A multi-objective stochastic programming model for emergency logistics based on goal programming. In 2011 Fourth International Joint Conference on Computational Sciences and Optimization, pages 640-644. IEEE, 2011. 


\section{A Appendix: Portland Metropolitan Dataset}

Refer tables A.1 and A.2 for list of demand points and potential facility locations from the Portland Metropolitan Dataset.

Table A.1: List of demand points with their location and population.

\begin{tabular}{|c|c|c|c|}
\hline ZCTA & Latitude & Longitude & Population \\
\hline 97014 & 45.5829 & -122.0168 & 0 \\
\hline 97019 & 45.5156 & -122.2427 & 362 \\
\hline 97024 & 45.5466 & -122.4424 & 2440 \\
\hline 97030 & 45.5092 & -122.4336 & 9291 \\
\hline 97060 & 45.5313 & -122.3691 & 2685 \\
\hline 97080 & 45.4783 & -122.3907 & 4027 \\
\hline 97201 & 45.5079 & -122.6908 & 3622 \\
\hline 97202 & 45.4827 & -122.6444 & 3942 \\
\hline 97203 & 45.6035 & -122.7379 & 6531 \\
\hline 97204 & 45.5184 & -122.6739 & 1663 \\
\hline 97205 & 45.5206 & -122.7102 & 458 \\
\hline 97206 & 45.4824 & -122.5986 & 5291 \\
\hline 97208 & 45.5287 & -122.6790 & 1482 \\
\hline 97209 & 45.5311 & -122.6839 & 2847 \\
\hline 97210 & 45.5442 & -122.7267 & 0 \\
\hline 97211 & 45.5811 & -122.6373 & 2436 \\
\hline 97212 & 45.5442 & -122.6435 & 2583 \\
\hline 97213 & 45.5382 & -122.6000 & 2647 \\
\hline 97214 & 45.5147 & -122.6430 & 3155 \\
\hline 97215 & 45.5151 & -122.6006 & 3299 \\
\hline 97216 & 45.5139 & -122.5584 & 6893 \\
\hline 97217 & 45.6018 & -122.7008 & 2198 \\
\hline 97218 & 45.5763 & -122.6009 & 3887 \\
\hline 97219 & 45.4542 & -122.6985 & 3185 \\
\hline \multicolumn{4}{|c|}{ Continued on next page } \\
\hline
\end{tabular}


TableA.1 - continued from previous page

\begin{tabular}{cccc}
\hline ZCTA & Latitude & Longitude & Population \\
\hline 97220 & 45.5500 & -122.5593 & 3164 \\
97221 & 45.4983 & -122.7288 & 954 \\
97227 & 45.5434 & -122.6781 & 4516 \\
97230 & 45.5578 & -122.5053 & 1847 \\
97231 & 45.6876 & -122.8242 & 221 \\
97232 & 45.5289 & -122.6439 & 2194 \\
97233 & 45.5151 & -122.5033 & 17964 \\
97236 & 45.4829 & -122.5098 & 6244 \\
97239 & 45.4924 & -122.6925 & 2214 \\
97266 & 45.4830 & -122.5582 & 6596 \\
97004 & 45.2550 & -122.4494 & 0 \\
97009 & 45.4230 & -122.3328 & 1129 \\
97011 & 45.3871 & -122.0264 & 510 \\
97013 & 45.2208 & -122.6683 & 2177 \\
97015 & 45.4135 & -122.5368 & 1726 \\
97017 & 45.1765 & -122.3897 & 536 \\
97022 & 45.3467 & -122.3200 & 940 \\
97023 & 45.2785 & -122.3232 & 952 \\
97027 & 45.3856 & -122.5928 & 4180 \\
97028 & 45.2884 & -121.8074 & 0 \\
97034 & 45.4094 & -122.6835 & 649 \\
97035 & 45.4135 & -122.7252 & 1575 \\
97038 & 45.0954 & -122.5590 & 1610 \\
97042 & 45.2052 & -122.5398 & 513 \\
97045 & 45.3203 & -122.5365 & 2057 \\
97049 & 45.3464 & -121.8624 & 0 \\
97055 & 45.3888 & -122.1552 & 538 \\
97067 & 45.2978 & -122.0544 & 32 \\
97068 & 45.3523 & -122.6686 & 1485 \\
97070 & 45.3061 & -122.7731 & 2451 \\
97086 & 45.4452 & -122.5281 & 985 \\
\hline & & Continued on next page \\
\hline & & &
\end{tabular}


TableA.1 - continued from previous page

\begin{tabular}{cccc}
\hline ZCTA & Latitude & Longitude & Population \\
\hline 97089 & 45.4266 & -122.4431 & 674 \\
97222 & 45.4409 & -122.6181 & 4847 \\
97267 & 45.4084 & -122.6129 & 3773 \\
98601 & 45.9434 & -122.3625 & 520 \\
98604 & 45.8057 & -122.5108 & 2335 \\
98606 & 45.7297 & -122.4564 & 805 \\
98607 & 45.6422 & -122.3800 & 634 \\
98629 & 45.8766 & -122.6192 & 997 \\
98642 & 45.8077 & -122.6939 & 745 \\
98660 & 45.6790 & -122.7205 & 0 \\
98661 & 45.6401 & -122.6250 & 7311 \\
98662 & 45.6885 & -122.5778 & 5303 \\
98663 & 45.6574 & -122.6632 & 3600 \\
98664 & 45.6195 & -122.5772 & 5072 \\
98665 & 45.6795 & -122.6606 & 3136 \\
98675 & 45.8285 & -122.3429 & 0 \\
98682 & 45.6732 & -122.4817 & 1008 \\
98683 & 45.6033 & -122.5102 & 2207 \\
98684 & 45.6306 & -122.5148 & 4580 \\
98685 & 45.7152 & -122.6931 & 1343 \\
98686 & 45.7234 & -122.6244 & 1651 \\
97016 & 46.0603 & -123.2670 & 905 \\
97018 & 45.8971 & -122.8106 & 1986 \\
97048 & 46.0448 & -122.9820 & 574 \\
97051 & 45.8793 & -122.9500 & 0 \\
97053 & 45.8280 & -122.8833 & 1800 \\
97054 & 45.9422 & -122.9496 & 453 \\
97056 & 45.7720 & -122.9694 & 0 \\
97064 & 45.8591 & -123.2355 & 385 \\
97101 & 45.0902 & -123.2287 & 743 \\
97111 & 45.2845 & -123.1952 & 4490 \\
\hline & & Continued on \\
& & & \\
& & & \\
& & \\
9 &
\end{tabular}


TableA.1 - continued from previous page

\begin{tabular}{cccc}
\hline ZCTA & Latitude & Longitude & Population \\
\hline 97114 & 45.1879 & -123.0766 & 560 \\
97115 & 45.2752 & -123.0395 & 926 \\
97127 & 45.2461 & -123.1114 & 576 \\
97128 & 45.2119 & -123.2822 & 1545 \\
97132 & 45.3242 & -122.9873 & 3811 \\
97148 & 45.3584 & -123.2485 & 0 \\
97347 & 45.0771 & -123.6564 & 0 \\
97396 & 45.1040 & -123.5490 & 848 \\
97005 & 45.4910 & -122.8036 & 5637 \\
97006 & 45.5170 & -122.8598 & 12425 \\
97007 & 45.4543 & -122.8796 & 3590 \\
97008 & 45.4602 & -122.8042 & 6272 \\
97062 & 45.3693 & -122.7623 & 2746 \\
97106 & 45.6657 & -123.1190 & 84 \\
97109 & 45.7378 & -123.1812 & 0 \\
97113 & 45.4972 & -123.0443 & 4865 \\
97116 & 45.5808 & -123.1657 & 926 \\
97117 & 45.6314 & -123.2884 & 0 \\
97119 & 45.4689 & -123.2002 & 84 \\
97123 & 45.4402 & -122.9801 & 297 \\
97124 & 45.5698 & -122.9496 & 6710 \\
97125 & 45.6711 & -123.1969 & 169 \\
97133 & 45.6861 & -123.0227 & 0 \\
97140 & 45.3531 & -122.8659 & 726 \\
97144 & 45.7416 & -123.3002 & 0 \\
97223 & 45.4403 & -122.7766 & 3395 \\
97224 & 45.4055 & -122.7951 & 3250 \\
97225 & 45.5016 & -122.7700 & 2053 \\
97229 & 45.5510 & -122.8093 & 2586 \\
98605 & 45.7769 & -121.6655 & 278 \\
98610 & 45.8659 & -122.0652 & 20 \\
\hline & & Continued on \\
& & & \\
& & \\
9 & \\
9 &
\end{tabular}


TableA.1 - continued from previous page

\begin{tabular}{cccc}
\hline ZCTA & Latitude & Longitude & Population \\
\hline 98616 & 46.1933 & -122.1329 & 0 \\
98639 & 45.6699 & -121.9897 & 0 \\
98648 & 45.7063 & -121.9563 & 808 \\
98651 & 45.7399 & -121.5835 & 0 \\
98671 & 45.6144 & -122.2384 & 446 \\
\hline \hline
\end{tabular}

Table A.2: List of all potential facility locations.

\begin{tabular}{ccc}
\hline ID & Latitude & Longitude \\
\hline 0 & 45.816915 & -122.745904 \\
1 & 45.862462 & -122.660465 \\
2 & 45.781175 & -122.527274 \\
3 & 45.691966 & -122.545208 \\
4 & 45.661016 & -122.636015 \\
5 & 45.625946 & -122.536095 \\
6 & 45.633546 & -122.661322 \\
7 & 45.643123 & -122.62553 \\
8 & 45.581972 & -122.390494 \\
9 & 45.579675 & -122.354185 \\
10 & 45.692315 & -121.894119 \\
11 & 45.737978 & -121.538641 \\
12 & 45.468176 & -123.143827 \\
13 & 45.598709 & -122.99608 \\
14 & 45.299255 & -122.974962 \\
15 & 45.304587 & -122.936475 \\
16 & 45.074026 & -123.614063 \\
17 & 45.212504 & -123.194003 \\
18 & 45.243158 & -123.116017 \\
19 & 45.275328 & -123.013592 \\
\hline & & Continued on next page \\
\hline
\end{tabular}


TableA.2 - continued from previous page

\begin{tabular}{|c|c|c|}
\hline ID & Latitude & Longitude \\
\hline 20 & 45.219097 & -123.07598 \\
\hline 21 & 45.097883 & -123.396 \\
\hline 22 & 45.076501 & -123.483198 \\
\hline 23 & 46.103447 & -123.202116 \\
\hline 24 & 46.09329 & -122.943955 \\
\hline 25 & 45.862614 & -122.808992 \\
\hline 26 & 45.845853 & -122.825924 \\
\hline 27 & 45.537161 & -122.265823 \\
\hline 28 & 45.285214 & -122.336807 \\
\hline 29 & 45.042412 & -122.667712 \\
\hline 30 & 45.149914 & -122.577736 \\
\hline 31 & 45.534584 & -122.624656 \\
\hline 32 & 45.570834 & -122.609652 \\
\hline 33 & 45.553662 & -122.675926 \\
\hline 34 & 45.533931 & -122.685373 \\
\hline 35 & 45.483126 & -122.804126 \\
\hline 36 & 45.516737 & -122.607121 \\
\hline 37 & 45.562518 & -122.666622 \\
\hline 38 & 45.598603 & -122.785298 \\
\hline 39 & 45.566168 & -122.674643 \\
\hline 40 & 45.550222 & -122.661925 \\
\hline 41 & 45.507636 & -122.421495 \\
\hline 42 & 45.520641 & -123.104218 \\
\hline 43 & 45.505128 & -122.485559 \\
\hline 44 & 45.426612 & -122.606107 \\
\hline 45 & 45.43116 & -122.582194 \\
\hline 46 & 45.477062 & -122.704034 \\
\hline 47 & 45.491318 & -122.601435 \\
\hline 48 & 45.303592 & -122.75862 \\
\hline 49 & 45.382808 & -122.732116 \\
\hline 50 & 45.38628 & -122.767007 \\
\hline \multicolumn{3}{|c|}{ Continued on next page } \\
\hline
\end{tabular}


TableA.2 - continued from previous page

\begin{tabular}{|c|c|c|}
\hline ID & Latitude & Longitude \\
\hline 51 & 45.385564 & -122.760503 \\
\hline 52 & 45.423316 & -122.768484 \\
\hline 53 & 45.536846 & -122.435763 \\
\hline 54 & 45.52117 & -123.057904 \\
\hline 55 & 45.53678 & -122.834329 \\
\hline 56 & 45.396788 & -122.269086 \\
\hline 57 & 45.44495 & -122.795228 \\
\hline 58 & 45.529033 & -122.8065 \\
\hline 59 & 45.252484 & -122.686501 \\
\hline 60 & 45.598734 & -122.996086 \\
\hline 61 & 45.352466 & -122.598023 \\
\hline 62 & 45.381646 & -122.592285 \\
\hline 63 & 45.44613 & -122.619198 \\
\hline 64 & 45.285227 & -122.336833 \\
\hline 65 & 45.383843 & -122.597554 \\
\hline 66 & 45.597585 & -123.001208 \\
\hline 67 & 45.562264 & -122.667592 \\
\hline 68 & 45.466562 & -122.753479 \\
\hline 69 & 45.522859 & -122.982042 \\
\hline 70 & 45.36081 & -122.845148 \\
\hline 71 & 45.431575 & -122.715113 \\
\hline 72 & 45.353494 & -122.866459 \\
\hline 73 & 45.409146 & -122.796286 \\
\hline 74 & 45.482443 & -122.588652 \\
\hline 75 & 45.440127 & -122.836567 \\
\hline 76 & 45.507387 & -122.797111 \\
\hline 77 & 45.569566 & -122.67361 \\
\hline 78 & 45.46818 & -122.709993 \\
\hline 79 & 45.494571 & -122.630546 \\
\hline 80 & 45.508503 & -122.717893 \\
\hline 81 & 45.149984 & -122.577833 \\
\hline \multicolumn{3}{|c|}{ Continued on next page } \\
\hline
\end{tabular}


TableA.2 - continued from previous page

\begin{tabular}{|c|c|c|}
\hline ID & Latitude & Longitude \\
\hline 82 & 45.519883 & -122.624607 \\
\hline 83 & 45.550336 & -122.685718 \\
\hline 84 & 45.542171 & -122.664863 \\
\hline 85 & 45.475815 & -122.722125 \\
\hline 86 & 45.44866 & -122.806375 \\
\hline 87 & 45.561751 & -122.681869 \\
\hline 88 & 45.35486 & -122.605961 \\
\hline 89 & 45.515352 & -122.974841 \\
\hline 90 & 45.470952 & -122.678958 \\
\hline 91 & 45.514686 & -122.976902 \\
\hline 92 & 45.527798 & -122.709669 \\
\hline 93 & 45.58773 & -122.711377 \\
\hline 94 & 45.515807 & -122.553233 \\
\hline 95 & 45.526807 & -122.578529 \\
\hline 96 & 45.464824 & -122.651252 \\
\hline 97 & 45.592235 & -122.75215 \\
\hline 98 & 45.479782 & -122.618847 \\
\hline 99 & 45.42624 & -122.667851 \\
\hline 100 & 45.369921 & -122.648819 \\
\hline 101 & 45.479756 & -122.804003 \\
\hline 102 & 45.539572 & -122.962627 \\
\hline 103 & 45.310153 & -122.798166 \\
\hline
\end{tabular}




\section{B Appendix: Standard P-Median Test Cases}

The OR-Library on J.E.Beasley website provides about twenty standard pmedian test cases with capacitated facilities. In all these test cases, the demand points themselves act as potential facility locations. These test cases can be accessed through: http://people.brunel.ac.uk/ mastjjb/jeb/orlib/ pmedcapinfo.html

Details of the two standard test cases used in this work are provided in this section. Table B.1 provides the coordinates and demand values of 50 sites in the first test case. Tables B.2 provides the same for 100 sites in the second test case. No. of facilities to open from the first and second test cases are 5 and 10 respectively. The capacity of each open facility is given as 120 .

Table B.1: List of demand-facility sites in test case-1 with their locations and demand values.

\begin{tabular}{cccc}
\hline ID & X-Coordinate & Y-Coordinate & Demand \\
\hline 1 & 2 & 62 & 3 \\
2 & 80 & 25 & 14 \\
3 & 36 & 88 & 1 \\
4 & 57 & 23 & 14 \\
5 & 33 & 17 & 19 \\
6 & 76 & 43 & 2 \\
7 & 77 & 85 & 14 \\
8 & 94 & 6 & 6 \\
9 & 89 & 11 & 7 \\
10 & 59 & 72 & 6 \\
11 & 39 & 82 & 10 \\
12 & 87 & 24 & 18 \\
13 & 44 & 76 & 3 \\
14 & 2 & 83 & 6 \\
15 & 19 & 43 & 20 \\
\hline \multicolumn{4}{c}{} \\
& & Continued on next page \\
\hline & & &
\end{tabular}


TableB.1 - continued from previous page

\begin{tabular}{|c|c|c|c|}
\hline ID & X-Coordinate & Y-Coordinate & Demand \\
\hline 16 & 5 & 27 & 4 \\
\hline 17 & 58 & 72 & 14 \\
\hline 18 & 14 & 50 & 11 \\
\hline 19 & 43 & 18 & 19 \\
\hline 20 & 87 & 7 & 15 \\
\hline 21 & 11 & 56 & 15 \\
\hline 22 & 31 & 16 & 4 \\
\hline 23 & 51 & 94 & 13 \\
\hline 24 & 55 & 13 & 13 \\
\hline 25 & 84 & 57 & 5 \\
\hline 26 & 12 & 2 & 16 \\
\hline 27 & 53 & 33 & 3 \\
\hline 28 & 53 & 10 & 7 \\
\hline 29 & 33 & 32 & 14 \\
\hline 30 & 69 & 67 & 17 \\
\hline 31 & 43 & 5 & 3 \\
\hline 32 & 10 & 75 & 3 \\
\hline 33 & 8 & 26 & 12 \\
\hline 34 & 3 & 1 & 14 \\
\hline 35 & 96 & 22 & 20 \\
\hline 36 & 6 & 48 & 13 \\
\hline 37 & 59 & 22 & 10 \\
\hline 38 & 66 & 69 & 9 \\
\hline 39 & 22 & 50 & 6 \\
\hline 40 & 75 & 21 & 18 \\
\hline 41 & 4 & 81 & 7 \\
\hline 42 & 41 & 97 & 20 \\
\hline 43 & 92 & 34 & 9 \\
\hline 44 & 12 & 64 & 1 \\
\hline 45 & 60 & 84 & 8 \\
\hline 46 & 35 & 100 & 5 \\
\hline
\end{tabular}


TableB.1 - continued from previous page

\begin{tabular}{cccc}
\hline ID & X-Coordinate & Y-Coordinate & Demand \\
\hline 47 & 38 & 2 & 1 \\
48 & 9 & 9 & 7 \\
49 & 54 & 59 & 9 \\
50 & 1 & 58 & 2 \\
\hline \hline
\end{tabular}

Table B.2: List of demand-facility sites in test case- 2 with their locations and demand values.

\begin{tabular}{cccc}
\hline ID & X-Coordinate & Y-Coordinate & Demand \\
\hline 1 & 6 & 5 & 12 \\
2 & 36 & 29 & 7 \\
3 & 61 & 45 & 1 \\
4 & 97 & 80 & 11 \\
5 & 51 & 74 & 1 \\
6 & 11 & 81 & 3 \\
7 & 18 & 92 & 4 \\
8 & 28 & 89 & 18 \\
9 & 25 & 3 & 12 \\
10 & 98 & 60 & 17 \\
11 & 3 & 33 & 12 \\
12 & 84 & 21 & 13 \\
13 & 88 & 7 & 10 \\
14 & 37 & 31 & 8 \\
15 & 78 & 39 & 13 \\
16 & 59 & 91 & 17 \\
17 & 87 & 5 & 14 \\
18 & 5 & 41 & 19 \\
19 & 80 & 95 & 2 \\
20 & 7 & 53 & 1 \\
21 & 11 & 77 & 5 \\
\hline \multicolumn{4}{c}{ Continued on next page } \\
\hline & & 106 & \\
\hline
\end{tabular}


TableB.2 - continued from previous page

\begin{tabular}{|c|c|c|c|}
\hline ID & X-Coordinate & Y-Coordinate & Demand \\
\hline 22 & 25 & 7 & 8 \\
\hline 23 & 8 & 6 & 12 \\
\hline 24 & 52 & 12 & 19 \\
\hline 25 & 63 & 71 & 15 \\
\hline 26 & 33 & 2 & 15 \\
\hline 27 & 31 & 54 & 18 \\
\hline 28 & 69 & 29 & 3 \\
\hline 29 & 50 & 52 & 1 \\
\hline 30 & 21 & 30 & 12 \\
\hline 31 & 14 & 86 & 9 \\
\hline 32 & 33 & 25 & 6 \\
\hline 33 & 94 & 91 & 2 \\
\hline 34 & 42 & 1 & 18 \\
\hline 35 & 59 & 60 & 12 \\
\hline 36 & 36 & 69 & 19 \\
\hline 37 & 61 & 3 & 14 \\
\hline 38 & 88 & 96 & 15 \\
\hline 39 & 73 & 22 & 9 \\
\hline 40 & 72 & 69 & 12 \\
\hline 41 & 7 & 26 & 2 \\
\hline 42 & 38 & 24 & 2 \\
\hline 43 & 45 & 36 & 6 \\
\hline 44 & 99 & 52 & 7 \\
\hline 45 & 48 & 44 & 12 \\
\hline 46 & 70 & 76 & 18 \\
\hline 47 & 51 & 50 & 17 \\
\hline 48 & 53 & 40 & 18 \\
\hline 49 & 39 & 55 & 19 \\
\hline 50 & 47 & 73 & 19 \\
\hline 51 & 70 & 15 & 5 \\
\hline 52 & 50 & 15 & 13 \\
\hline \multicolumn{4}{|c|}{ Continued on next page } \\
\hline
\end{tabular}


TableB.2 - continued from previous page

\begin{tabular}{cccc}
\hline ID & X-Coordinate & Y-Coordinate & Demand \\
\hline 53 & 83 & 79 & 7 \\
54 & 67 & 4 & 18 \\
55 & 23 & 41 & 17 \\
56 & 41 & 85 & 1 \\
57 & 50 & 16 & 19 \\
58 & 83 & 64 & 9 \\
59 & 30 & 95 & 14 \\
60 & 47 & 39 & 12 \\
61 & 20 & 41 & 1 \\
62 & 97 & 76 & 4 \\
63 & 88 & 49 & 7 \\
64 & 54 & 97 & 2 \\
65 & 44 & 51 & 17 \\
66 & 14 & 19 & 19 \\
67 & 60 & 70 & 20 \\
68 & 83 & 52 & 13 \\
69 & 18 & 42 & 1 \\
70 & 71 & 39 & 6 \\
71 & 94 & 49 & 8 \\
72 & 28 & 8 & 12 \\
73 & 88 & 49 & 17 \\
74 & 33 & 60 & 5 \\
75 & 50 & 83 & 15 \\
76 & 57 & 14 & 18 \\
77 & 58 & 11 & 11 \\
78 & 57 & 87 & 7 \\
79 & 52 & 88 & 1 \\
80 & 89 & 83 & 2 \\
81 & 31 & 92 & 1 \\
82 & 26 & 2 & 10 \\
83 & 61 & 68 & 3 \\
\hline & & Continued on next page \\
\hline
\end{tabular}


TableB.2 - continued from previous page

\begin{tabular}{cccc}
\hline ID & X-Coordinate & Y-Coordinate & Demand \\
\hline 84 & 37 & 15 & 8 \\
85 & 60 & 41 & 17 \\
86 & 51 & 55 & 11 \\
87 & 56 & 20 & 5 \\
88 & 19 & 11 & 4 \\
89 & 21 & 95 & 9 \\
90 & 42 & 48 & 6 \\
91 & 48 & 89 & 11 \\
92 & 37 & 71 & 14 \\
93 & 6 & 31 & 6 \\
94 & 47 & 12 & 13 \\
95 & 2 & 58 & 4 \\
96 & 27 & 4 & 18 \\
97 & 28 & 54 & 16 \\
98 & 61 & 81 & 7 \\
99 & 79 & 91 & 10 \\
100 & 78 & 13 & 4 \\
\hline \hline
\end{tabular}

